\title{
40. PHYSICAL PROPERTIES OF SEDIMENTS OF THE COSTA RICA RIFT, DEEP SEA DRILLING PROJECT SITES 504 AND 5051
}

\author{
R. H. Wilkens, ${ }^{2}$ Department of Geological Sciences, University of Washington, Seattle, Washington \\ and \\ M. G. Langseth, Lamont-Doherty Geological Observatory, Palisades, New York
}

\begin{abstract}
Values of physical properties measured in the upper sections of sediment cores recovered at Sites 504 and 505 exhibit a remarkable similarity. Below a depth of $145 \mathrm{~m}$ Site 504 sediments appear to have undergone changes which are reflected in physical property values. This alteration may have been due to high temperatures in the sediment. In most of Site 505 , and in Site 504 above $145 \mathrm{~m}$, seismic velocity averages $1.51 \mathrm{~km} / \mathrm{s}$, wet bulk density $1.32 \mathrm{~g} / \mathrm{cm}^{3}$, porosity $80 \%$, and thermal conductivity $0.80 \% \mathrm{~W} / \mathrm{m}{ }^{\circ} \mathrm{K}$. Below $145 \mathrm{~m}$ at Site 504 and $210 \mathrm{~m}$ at Site 505 , mean density increases to $1.40 \mathrm{~g} / \mathrm{cm}^{3}$, porosity decreases to $67 \%$, seismic velocity increases to $1.53 \mathrm{~km} / \mathrm{s}$, and thermal conductivity increases to values in excess of $1.0 \mathrm{~W} / \mathrm{m}{ }^{\circ} \mathrm{K}$. A good correlation between independent measurements of water content and thermal resistivity supports the existence of small but regular variation in the measured parameters on the scale of $10 \mathrm{~m}$ and less.
\end{abstract}

\section{INTRODUCTION}

A comprehensive program of physical-properties measurements was carried out on sediments recovered from Holes $504\left(1^{\circ} 13.6^{\prime} \mathrm{N}, 83^{\circ} 43.9^{\prime} \mathrm{W}\right)$ and $505\left(1^{\circ} 54.82^{\prime} \mathrm{N}\right.$, $83^{\circ} 47.39^{\prime} \mathrm{W}$ ) of Leg 69 . Investigated were compressional-wave seismic velocity, bulk density, grain density, porosity and water content, thermal conductivity, and, for Hole 504 only, shear strength. Data from all measurements for Holes 504 and 505 are presented in Table 1.

Comparison of data from the two sites is interesting for several reasons. Because the sites are relatively close (504 is $80 \mathrm{~km}$ south of 505), the sediments are very similar. Sediments recovered from both holes are mainly carbonatic siliceous oozes with variable proportions of clay. Diagenesis is more advanced at Site 504, owing to a higher temperature gradient, which has resulted in the formation of chert near the bottom of the sediment column; this has not occurred at Site 505. Sediment recovery down to the cherts in Hole 504 was accomplished with a new DSDP tool, the hydraulic piston corer, whereas the sediments at Hole 505 were cored in the standard fashion, by rotary drilling. At Site 504, conventional coring was used to recover materials below the topmost cherts, down to basaltic basement (Holes 501, 504A, and 504B).

The close agreement of values measured at each site in time-stratigraphically equivalent cores demonstrates that, with careful sample selection, the effects of drilling disturbance on physical-properties measurements is minimal. In cores from deeper levels, average values from the two sites begin to diverge. This is a result of the differing thermal regimes at the sites (see Langseth et

\footnotetext{
${ }^{1}$ Cann, J. R., Langseth, M. G., Honnorez, J., Von Herzen, R. P., White, S. M., et al., Init. Repts. DSDP, 69: Washington (U.S. Govt. Printing Office).

2 Present address: Department of Earth and Planetary Sciences, Massachusetts Institute of Technology, Cambridge, Massachusetts.
}

al., this volume), and the contrasting diagenetic effects mentioned earlier. Finally, good correlation of independent measurements such as thermal resistivity and water content supports the existence in each sediment column of a fine-scale structure that varies on the order of meters.

\section{MEASUREMENT PROCEDURES}

Cores were kept unopened in the laboratory for several hours to ensure that they were nearly equilibrated to laboratory temperature. After equilibration, thermal-conductivity measurements were made by the standard needle-probe technique (Von Herzen and Maxwell, 1959). The needle was inserted in a small hole drilled in the unopened liner. After this determination, the core was split, and the working half was given over to the rest of the physical-properties procedures. Penetrometer and vane shear measurements were made according to the procedures in Boyce (1977). The penetrometer experiment is performed by dropping a needle from a height of $1 \mathrm{~cm}$ above the sediment and recording the depth of penetration. Vane shear measurements were made by applying increasing torque at a constant rate to a four-bladed vane embedded in the sediment. Shear strength is then calculated from the amount of torque applied to the vane at the time of failure of the sediment.

Seismic-velocity measurements were made with the Hamilton frame velocimeter, following the procedures of Boyce (1976b). Prior to sediment analysis, all operators of the velocimeter performed repeated runs on standards of brass, aluminum, and lucite. Obtained velocities were consistently within $1 \%$ of standard values, so no correction was applied for instrumental or operator bias. Because most of the sediment was very loosely consolidated, all measurements were made with the sediment in the half liner, which means that all reported velocities are in the originally horizontal plane. Corrections for core-liner thickness and time delay were determined prior to the analysis and applied to the raw data.

A sample of approximately $10 \mathrm{~cm}^{3}$ volume was taken for gravimetric analysis, following the procedures of Boyce (1976). Samples were weighed wet in air, submerged in water, and, finally, after drying for 24 hours in an oven at $100^{\circ} \mathrm{C}$. Determinations of wet-bulk density and water content were made, from which grain density and porosity were derived. Salt-water corrections were applied in the calculation of final values.

The excellence of the hydraulic piston corer in returning a high percentage of the sediments cored in a virtually undisturbed state allowed measurement and sampling at Hole 504 on almost every section of core, resulting in a sampling interval of approximately 1.5 meters. Drilling disturbance at Site 505 precluded such detailed ex- 
amination. By sampling only in areas that appeared relatively undisturbed, the sampling interval was made somewhat variable, averaging about 3 meters.

\section{SEISMIC VELOCITY}

Seismic velocity exhibits the least variation with depth of any of the parameters measured (Fig. 1). The data from Hole 505 vary around a mean of $1.51 \mathrm{~km} / \mathrm{s}$ from the top to the bottom of the hole. The velocity in the upper section of Hole 504, from 10 to 145 meters, varies around a similar mean, although the dense sampling and undisturbed nature of the returned sediments supports the existence of regular, small-scale excursions from the $1.51-\mathrm{km} / \mathrm{s}$ mean compressional velocity. Below 145 meters, there is an abrupt increase in the mean seismic velocity to around $1.53 \mathrm{~km} / \mathrm{s}$. The shift is small, but supported by the data, and it corresponds to abrupt and more-apparent changes in other measured physical properties. This level also corresponds to a major break in the sedimentary lithology at this site (Beiersdorf and Natland, this volume). Velocities from both of the sites are in agreement with other determinations from unconsolidated siliceous nannofossil oozes recovered from DSDP sites (Hamilton, 1978). Two additional velocities were measured in atypical materials at Site 504. A 2-cmthick ash layer at 178 meters sub-bottom yielded a seismic velocity of $1.65 \mathrm{~km} / \mathrm{s}$, and a chert fragment from 232 meters in Hole 504A exhibited a velocity of 4.25 $\mathrm{km} / \mathrm{s}$.

\section{GRAVIMETRIC DATA}

The gravimetric data exhibit much greater change through the sediment column, and thus are more useful than velocity in making comparisons between holes or in looking at fine structure at each site. Wet-bulk density and porosity are plotted versus depth in Figure 1 and versus each other in Figure 2. Examining Hole 504 first, we can see that, in general, the values of wet-bulk density recorded for the upper interval of 10 to 45 meters sub-bottom are between 1.3 and $1.4 \mathrm{~g} / \mathrm{cm}^{3}$. There is one significant deviation from this range between 60 and 100 meters, where values drop to a minimum of 1.21 $\mathrm{g} / \mathrm{cm}^{3}$. Between 140 and 160 meters, values become somewhat more variable, and by 170 meters generally are scattered between 1.45 and $1.55 \mathrm{~g} / \mathrm{cm}^{3}$. A comparison of these values with those of Hole 505 shows good correlation. Bulk-density values in samples from the top of Hole 505 are somewhat less than those in Site 504, ranging from 1.25 to $1.35 \mathrm{~g} / \mathrm{cm}^{3}$. This may be a result of drilling disturbance of the most loosely consolidated part of the Hole 505 sediment column. The minimum, centered about 75 meters at Hole 504, occurs between 70 and 130 meters at Site 505, both minima agreeing in absolute value $\left(1.21 \mathrm{~g} / \mathrm{cm}^{3}\right)$ and in age of sediment. Lithologic data (Beiersdorf and Natland, this volume) suggest that these zones correspond to areas of minimum carbonate in the sediments.

What is not seen at the bottom of the sediment column at Hole 505 is the increase in variability of bulk densities seen at Hole 504. Sediments below approximately 170 meters at Hole 504 were deposited before formation of the oceanic basement of Hole 505 at a ridge crest $3.9 \mathrm{~m} . \mathrm{y}$. ago, but the variability begins at higher levels. Figure 2, wet-bulk density versus porosity, contains superimposed lines of calculated grain densities. Sediments from the upper unit of Hole 504 and all of Hole 505 generally fall about a grain density of 2.70 $\mathrm{g} / \mathrm{cm}^{3}$. Those from lower in the Hole 504 column are significantly less dense, suggesting perhaps a greater amount of hydration of the sediment particles due to the increase in temperature with depth, or the formation of lighter carbonate phases.

Porosity essentially mirrors bulk density, which is the expected relationship for most of the two sediment columns, where grain density is relatively constant. Porosity generally decreases with depth, because of burial compaction. Sediments at the base of Hole 504 attain lower porosities than those at Hole 505, but the concurrent decrease in grain density has kept the bulk density roughly the same as that at Hole 505 .

\section{THERMAL CONDUCTIVITY}

Thermal conductivity $(k)$ values (Fig. 3 ) have been corrected for in situ conditions, using the relationships of Ratcliffe (1960; see also Hyndman et al., 1974). At Hole 504, the profile of $k$ versus depth can be divided into two distinct zones, as for other physical properties. In the sub-bottom depth interval from 10 to 145 meters, conductivity gradually increases with depth from near $0.75 \mathrm{~W} / \mathrm{m}^{\circ} \mathrm{K}$ near the sea floor to $0.85 \mathrm{~W} / \mathrm{m}^{\circ} \mathrm{K}$ at a depth of 145 meters. Values near the top of the interval are in agreement with those measured in piston cores taken during the site survey (Langseth et al., this volume). In the interval from 145 to 235 meters, the $k$ values are significantly higher and exhibit a greater variability about a generally increasing trend.

Variations of thermal conductivity on a scale of 10 meters are observed in the upper unit. A local maximum at a depth of 60 meters is followed by a minimum centered at 75 meters, reflecting changes already described in the bulk-density and porosity profiles (Fig. 1). It is well established that the thermal conductivity of unconsolidated sea-floor sediment depends principally on water content. Thus, the measurements of $k$ provide an independent indication of the variation of water content with depth. The general agreement between the $k$ profile and the profiles of gravimetric data provides strong support that the small variations are real.

The sediments at Hole 505 show the same general pattern as at Hole 504, values in the upper 100 meters averaging $0.77 \mathrm{~W} / \mathrm{m}^{\circ} \mathrm{K}$, and those in the second 100 meters gradually increasing to near $0.87 \mathrm{~W} / \mathrm{m}^{\circ} \mathrm{K}$. Below 210 meters, values are significantly higher, averaging about $1.05 \mathrm{~W} / \mathrm{m}^{\circ} \mathrm{K}$ in sediments that are somewhat more consolidated.

The correlation between water content and thermal resistivity $(1 / k)$ is tested in Figure 4 . As in the densityporosity plot, most of the data from Hole 505 fall along the same trend as that from the upper unit of Hole 504. Data points with arrows indicate possible errors due to equipment problems and have not been included in the following regression calculations. A linear relationship 


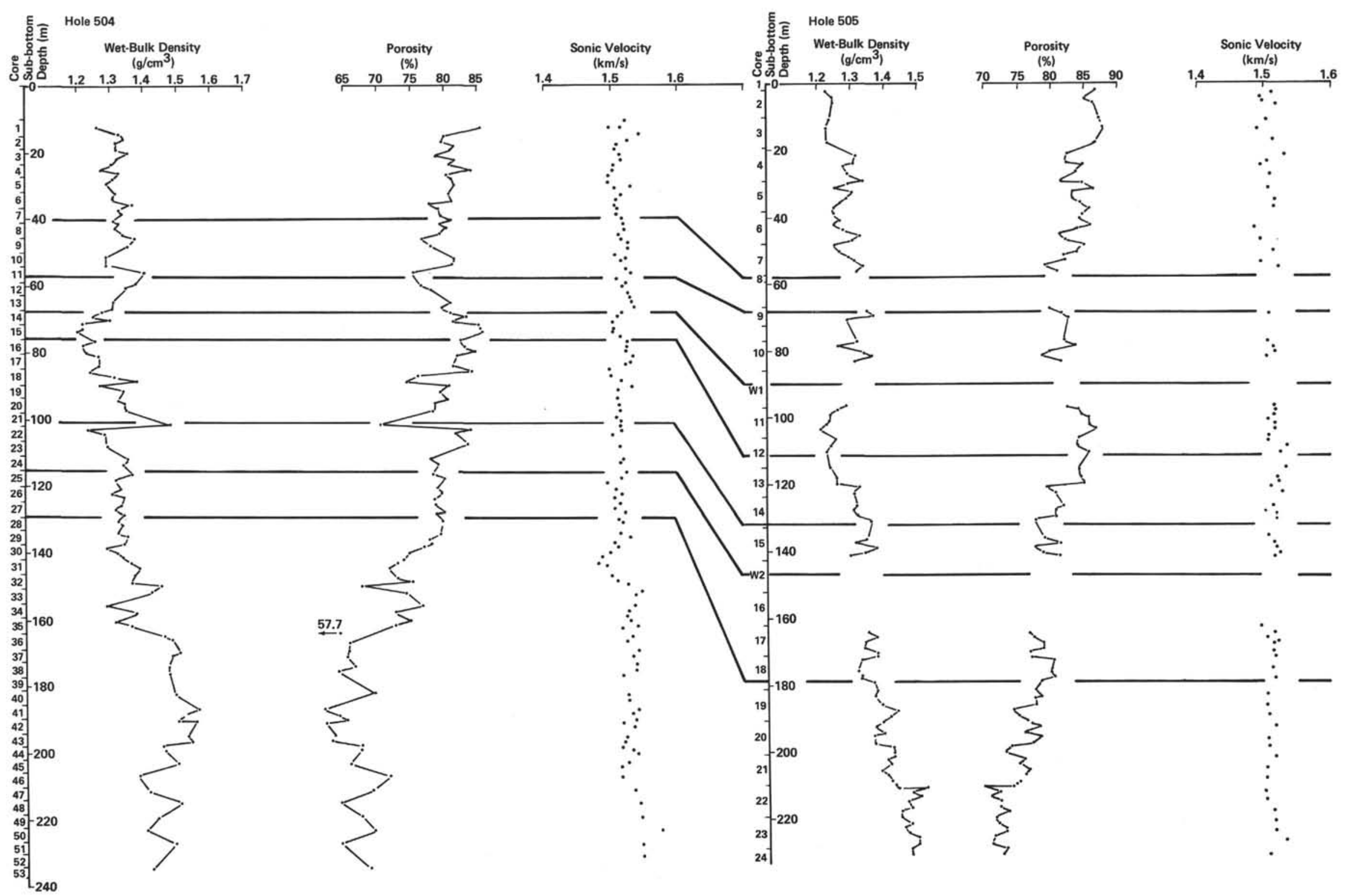

Figure 1. Wet-bulk density, porosity, and sonic velocity versus depth for sediments from Holes 504 and 505 . Lines connect depths of the same biostratigraphic age (Sancetta, this volume). 


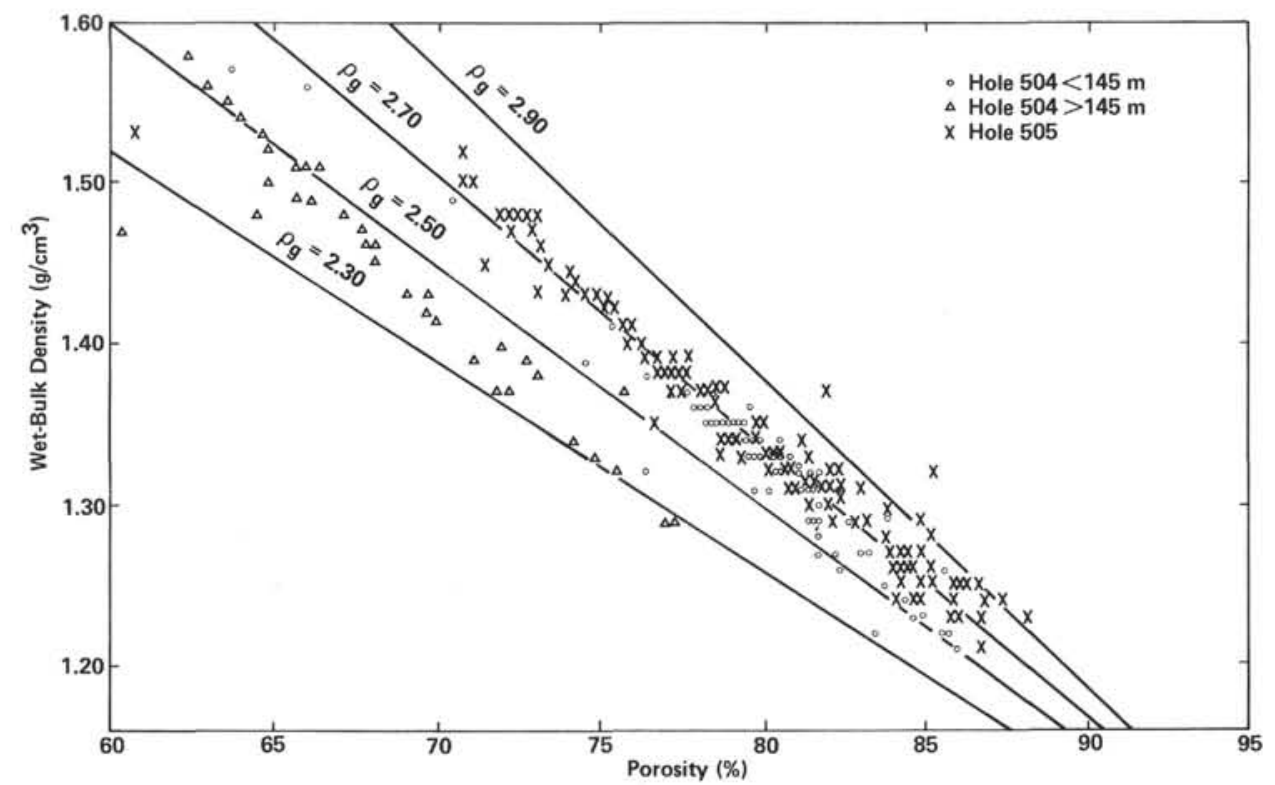

Figure 2. Porosity versus wet-bulk density for sediments from Holes 504 and 505 . Lines represent calculated grain-density relations.

seen in previous studies is indicated (Ratcliffe, 1960; Bullard, 1963; Lachenbruch and Marshall, 1968), and a line for all of the Hole 504 data was calculated, which gives the relation

$$
R=-0.1259+2.10 w, r^{2}=0.91,
$$

where $w$ is the proportion of water by wet weight. The high value of the correlation coefficient indicates a good fit; however, the relation is unsatisfactory, because it predicts a negative thermal resistivity at $0 \%$ porosity. Furthermore, it has a steep slope compared to those determined from other studies (for example, Bullard and Day, 1961; see Fig. 4). A more reasonable result is obtained if it is assumed that the data from 0 to 145 meters and 145 to 235 meters form two separate groups. The relations derived by linear regression are:

$$
R=0.459+1.25 w, r^{2}=0.44
$$

for unit I, and

$$
R=0.394+0.97 w, r^{2}=0.38
$$

for unit II. These relations are thought to be physically more realistic. One interpretation of this separation is that the sediments of unit II have formed better conductive links between grains and thus are less sensitive to changes in porosity. The deepest measurements made at Hole 505 fall in the general grouping of the lower Hole 504 values and probably reflect increasing importance of the thermal-conduction properties of the solid skeleton of the sediment as porosity decreases. Unfortunately, the low correlation coefficients for the two groups of data rule out any positive demonstration of this hypothesis.

\section{SHEAR STRENGTH}

Penetrability steadily decreases down Hole 504 (Fig. 5). There is perhaps a change in slope at approximately 55 meters that might indicate a change in the rate of compaction.

Values of shear strength (Fig. 5) at the top of the hole, 100 to $200 \mathrm{~g} / \mathrm{cm}^{2}$, are in agreement with measurements from deep-ocean piston cores of pelagic sediments. Below 100 meters, the scatter in the measurements increases greatly, but the trend continues to be that of increasing shear strength with depth. Maximum values of shear strength, $1600 \mathrm{~g} / \mathrm{cm}^{2}$, were recorded at approximately 170 meters. Below that depth, a rock saw was employed to split the cores. Greatly reduced values of shear strength in the bottom 60 meters of the hole are due to mechanical disruption during this core splitting, and should be disregarded.

\section{SUMMARY}

Physical-properties measurements at Holes 504 and 505 exhibit similarities in the upper regions of the sedimentary columns and differ toward their bases. The sediment at the base of Hole 504 underwent changes due to high temperatures (Hein and Yeh, this volume). The bulk of Hole 505 sediments and Hole 504 sediments to a depth of 145 meters have fairly uniform physical properties. In this interval, seismic velocity averages 1.51 $\mathrm{km} / \mathrm{s}$, wet-bulk density averages $1.32 \mathrm{~g} / \mathrm{cm}^{3}$, porosity averages $80 \%$, and thermal conductivity is close to 0.80 $\mathrm{W} / \mathrm{m}^{\circ} \mathrm{K}$. Below 145 meters at Hole 504 , and 210 meters at Hole 505 , mean density increases to $1.48 \mathrm{~g} / \mathrm{cm}^{3}$, porosity decreases to $67 \%$, seismic velocity increases to $1.53 \mathrm{~km} / \mathrm{s}$, and thermal conductivity increases to values in excess of $1.0 \mathrm{~W} / \mathrm{m}^{\circ} \mathrm{K}$. The good correlation between independent measurements of water content and ther- 


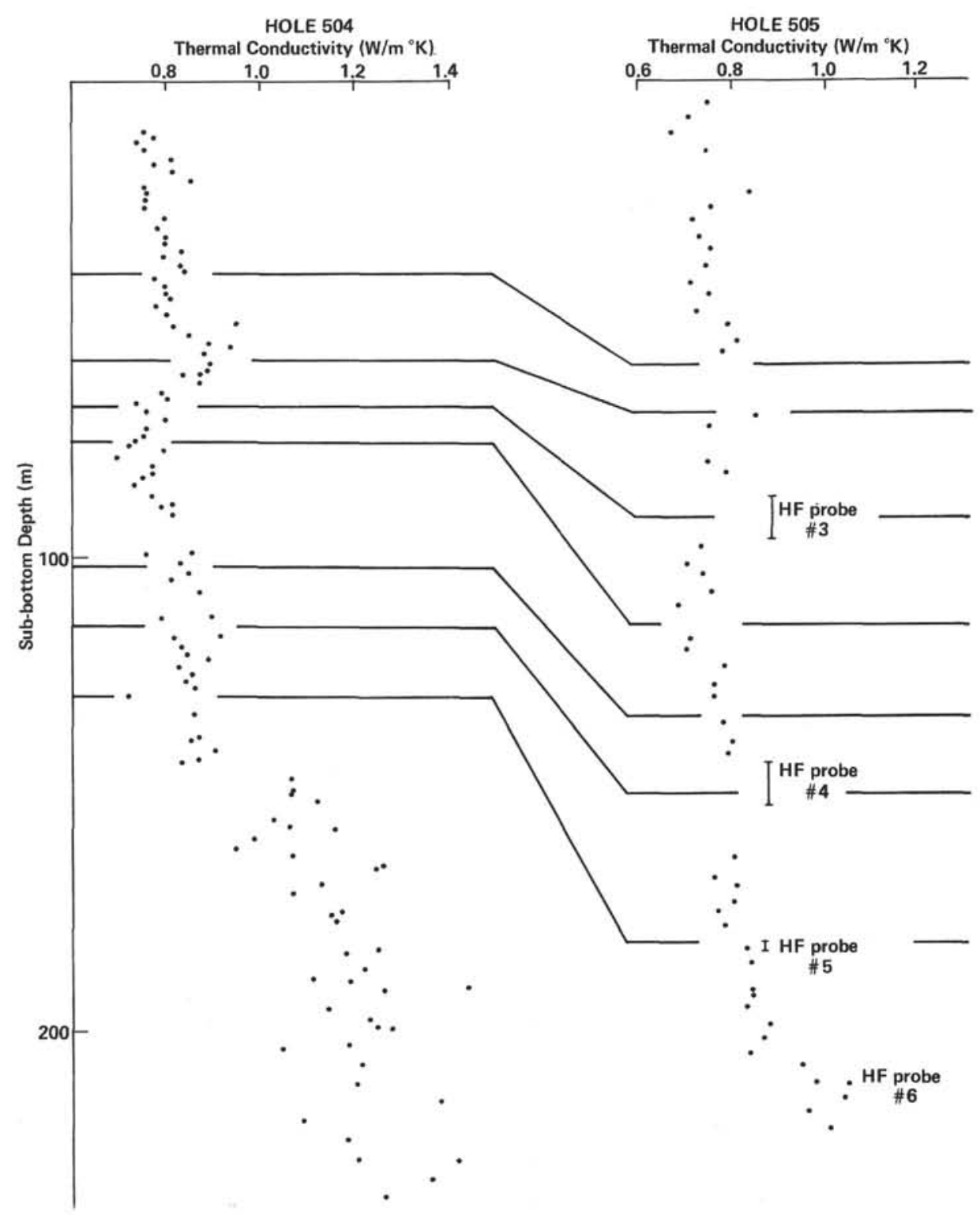

Figure 3. Thermal conductivity versus depth for sediments from Holes 504 and 505. Lines connect depths of the same biostratigraphic age (Sancetta, this volume).

mal resistivity supports the existence of small but regular variation in the measured parameters on a scale of 10 meters and less.

\section{ACKNOWLEDGMENTS}

Partial support for the cruise participation of R. H. Wilkens was provided by Office of Naval Research grant number N0014-80-C-0252 to Dr. N. I. Christensen. The participation of Marcus Langseth in this cruise was supported by a National Science Foundation grant OCE79-19387. K. Horai and D. Sawyer reviewed the manuscript, and their interest is appreciated.

\section{REFERENCES}

Boyce, R. E., 1976. Definitions and laboratory techniques of compressional sound velocity parameters and wet-water content, wetbulk density, and porosity parameters by gravimetric and gammaray attenuation techniques. In Schlanger, S. O., Jackson, E. D., et al., Init. Repts. DSDP, 33: Washington (U.S. Govt. Printing Office), 695-728.

1977. Deep Sea Drilling Project procedures for shear strength measurement of clay sediment using modified Wykeham
Farrance laboratory vane apparatus. In Barker, P. F., Dalziel, I. W. D., et al., Init. Repts. DSDP, 36: Washington (U.S. Govt. Printing Office), 1059-1068.

Bullard, E. C., 1963. The flow of heat through the floor of the ocean. In Hill, M. N. (Ed.), The Sea (Vol. 3): New York (Wiley-Interscience), 218.

Bullard, E. C., and Day, A., 1961. The flow of heat through the floor of the Atlantic Ocean. Geophys. J. Royal Astron. Soc., 4: 282-292.

Hamilton, E. L., 1978. Sound velocity-density relations in sea-floor sediments and rocks. J. Acoust. Soc. Am., 63:366-377.

Hyndman, R. D., Erickson, A. J., and Von Herzen, R. P., 1974. Geothermal measurements on DSDP Leg 26. In Davies, T. A., Luyendyk, B. P., et al., Init. Repts. DSDP, 26: Washington (U.S. Govt. Printing Office), 451-463.

Lachenbruch, A. H., and Marshall, B. V., 1968. Heat flow and water temperature fluctuations in the Denmark Strait. J. Geophys. Res., 73:5829-5842.

Ratcliffe, E. H., 1960. The thermal conductivities of ocean sediments. J. Geophys. Res., 65:1535.

Von Herzen, R. P., and Maxwell, A. E., 1959. The measurement of thermal conductivity of deep-sea sediments by a needle probe method. J. Geophys. Res., 64:1557. 


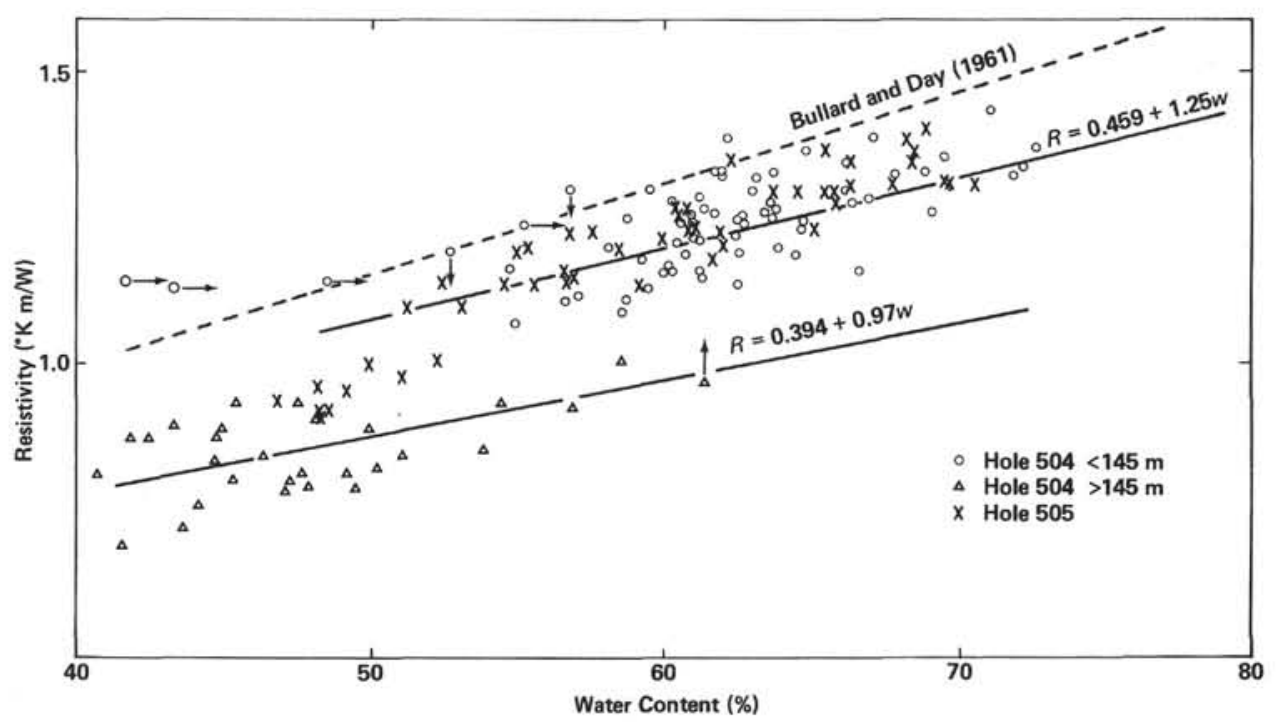

Figure 4. Thermal resistivity versus water content for sediments from Holes 504 and 505. Dashed line is the fit of Bullard and Day (1961) to their data. Solid lines are regression lines for Hole 504 data above and below 145 meters. 


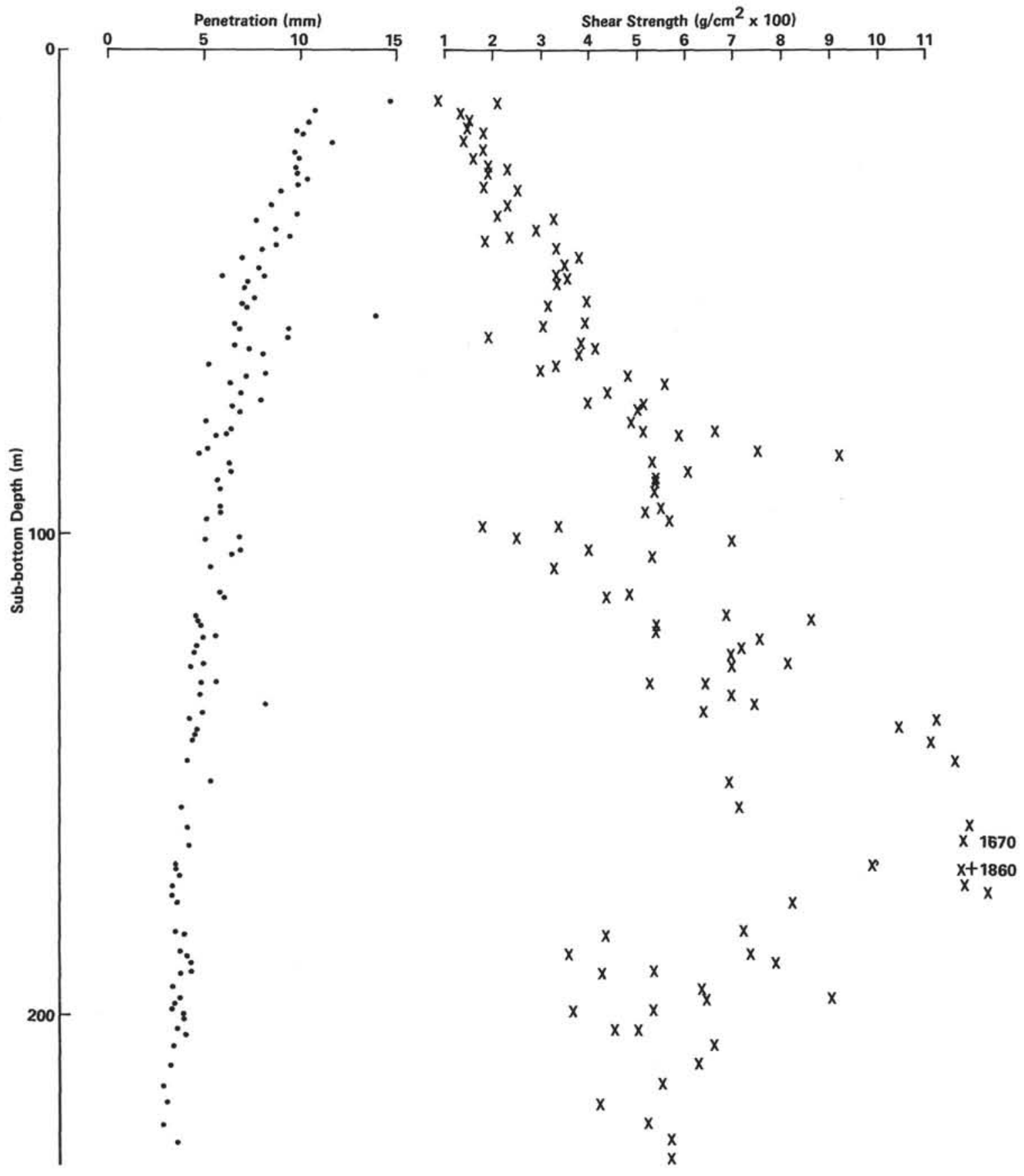

Figure 5. Shear-strength and penetrometer measurements versus depth for sediments from Hole 504. A rotary power saw was used to cut sediment cores below 170 meters. 


\section{R. H. WILKENS, M. G. LANGSETH}

Table 1. Physical properties of sediments, Holes 504 and 505 .

\begin{tabular}{|c|c|c|c|c|c|c|}
\hline $\begin{array}{c}\text { Sample } \\
\text { (level or interval in cm) }\end{array}$ & $\underset{\left(g / \mathrm{cm}^{3}\right)}{\mathrm{eb}}$ & $\underset{\left(g / \mathrm{cm}^{3}\right)}{\stackrel{e g}{3}}$ & $\begin{array}{c}\phi \\
(\%)\end{array}$ & $\begin{array}{l}\text { Water } \\
\text { Content } \\
\text { (wt. \%) }\end{array}$ & $\underset{\substack{V / p \\
(\mathrm{~km} / \mathrm{s})}}{V_{0}}$ & $\begin{array}{l}\text { Shear } \\
\text { Strength } \\
\left(\mathrm{g} / \mathrm{cm}^{2}\right)\end{array}$ \\
\hline $504-1-1,44$ & - & - & - & - & 1.52 & - \\
\hline $1-1,47$ & - & - & - & - & 1.52 & - \\
\hline $1-1,52$ & - & - & - & - & - & 85 \\
\hline $1-1,104$ & - & - & - & - & - & - \\
\hline $1-2,94$ & - & - & - & - & - & 208 \\
\hline $1-2,98$ & - & - & - & - & - & - \\
\hline $1-2,103-105$ & 1.26 & 2.64 & 85.7 & 70.0 & - & - \\
\hline $1-3,46$ & - & - & - & - & - & 125 \\
\hline $1-3,53$ & - & $\overline{-}$ & $=$ & - & 1.51 & - \\
\hline $1-3,61$ & - & - & - & - & - & - \\
\hline $2-1,41$ & - & - & - & - & - & - \\
\hline $2-1,73-75$ & 1.33 & 2.55 & 80.0 & 61.7 & - & - \\
\hline $2-1,79$ & - & - & - & - & 1.54 & - \\
\hline $2-1,105$ & - & - & - & - & - & 146 \\
\hline $2-2,53$ & - & - & - & - & - & - \\
\hline $2-2,73-75$ & 1.34 & 2.58 & 79.8 & 61.0 & - & - \\
\hline $2-2,84$ & - & - & - & - & - & 148 \\
\hline $2-2,92$ & - & - & - & $\overline{-}$ & 1.53 & - \\
\hline $2-3,33$ & - & - & - & - & - & - \\
\hline $2-3,43$ & - & - & - & - & - & 179 \\
\hline $2-3,56$ & - & - & - & - & 1.51 & - \\
\hline $2-3,63-65$ & 1.32 & 2.60 & 81.2 & 63.0 & - & - \\
\hline $3-1,45$ & - & - & - & - & - & - \\
\hline $3-1,73-75$ & 1.32 & 2.60 & 81.0 & 62.7 & - & - \\
\hline $3-1,81$ & - & - & - & - & $\overline{-}$ & 135 \\
\hline $3-1,95$ & - & - & - & - & 1.51 & - \\
\hline $3-2,40$ & $\overline{-}$ & - & - & $\overline{-}$ & - & - \\
\hline $3-2,73-75$ & 1.36 & 2.62 & 78.8 & 59.2 & - & - \\
\hline $3-2,80$ & - & - & - & - & - & 184 \\
\hline $3-2,109$ & - & - & - & - & 1.52 & - \\
\hline $3-3,40$ & - & - & - & - & - & - \\
\hline $3-3,69$ & - & - & - & - & - & 159 \\
\hline $3-3,73-75$ & 1.32 & 2.64 & 81.8 & 63.7 & - & - \\
\hline $3-3,94$ & - & - & - & - & 1.52 & - \\
\hline $4-1,40$ & $=$ & $\overline{-}$ & $\bar{z}$ & $\bar{z}$ & - & $\overline{-}$ \\
\hline $4-1,73-75$ & 1.31 & 2.53 & 80.8 & 63.1 & - & - \\
\hline $4-1,89$ & - & - & - & - & - & 188 \\
\hline $4-1,108$ & - & - & - & - & 1.52 & - \\
\hline $4-2,39$ & - & - & - & - & - & - \\
\hline $4-2,57$ & - & - & - & - & - & 233 \\
\hline $4-2,73-75$ & 1.27 & 2.59 & 84.3 & 68.0 & - & - \\
\hline $4-2,109$ & - & - & - & - & 1.51 & - \\
\hline $4-3,41$ & - & - & - & - & - & - \\
\hline $4-3,55$ & - & - & - & - & - & 191 \\
\hline $4-3,73-75$ & 1.33 & 2.58 & 80.4 & 62.0 & - & - \\
\hline $4-3,81$ & - & - & - & - & 1.51 & - \\
\hline $5-1,50$ & - & - & - & - & - & - \\
\hline $5-1,69$ & - & - & - & - & - & 175 \\
\hline $5-1,73-75$ & 1.31 & 2.55 & 81.4 & 63.8 & - & - \\
\hline $5-1,105$ & - & - & - & - & 1.50 & - \\
\hline $5-2,76$ & - & - & - & - & - & 251 \\
\hline $5-2,83-85$ & 1.29 & 2.50 & 81.7 & 64.7 & $=$ & - \\
\hline $5-3,103$ & - & - & - & - & 1.50 & $=$ \\
\hline $5-3,27$ & - & - & - & - & - & - \\
\hline $5-3,37$ & - & - & - & - & 1.53 & - \\
\hline $6-1,40$ & - & - & - & - & - & - \\
\hline $6-1,63$ & - & - & - & - & - & 229 \\
\hline $6-1,73-75$ & 1.32 & 2.57 & 80.0 & 62.7 & - & - \\
\hline $6-1,100$ & - & - & - & - & 1.52 & - \\
\hline $6-2,43$ & - & - & - & - & - & - \\
\hline $6-2,73-75$ & 1.31 & 2.57 & 81.3 & 63.5 & - & - \\
\hline $6-2,94$ & - & - & - & - & $=$ & $\overline{214}$ \\
\hline $6-2,106$ & - & - & - & - & 1.51 & - \\
\hline $6-3,44$ & - & - & - & - & - & - \\
\hline $6-3,53$ & - & - & - & - & - & 331 \\
\hline $6-3,73-75$ & 1.37 & 2.59 & 77.8 & 58.1 & - & - \\
\hline $6-3,100$ & - & - & - & - & 1.51 & - \\
\hline $7-1,47$ & - & - & - & - & - & - \\
\hline $7-1,73-75$ & 1.33 & 2.53 & 79.6 & 61.3 & - & - \\
\hline $7-1,39$ & - & - & - & - & - & 285 \\
\hline $7-1,104$ & $=$ & $\bar{z}$ & $=$ & $=$ & 1.51 & - \\
\hline $7-2,42$ & - & - & - & - & - & - \\
\hline $7-2,58$ & - & - & - & $=$ & $=$ & 230 \\
\hline $7-2,73-75$ & 1.34 & 2.60 & 79.7 & 60.8 & - & - \\
\hline $7-2,100$ & - & - & - & - & 1.51 & - \\
\hline $7-3,39$ & - & - & - & - & - & - \\
\hline $7-3,60$ & - & - & - & - & - & 182 \\
\hline $7-3,73-75$ & 1.31 & 2.57 & 81.7 & 63.9 & - & - \\
\hline $7-3,116$ & - & - & - & - & 1.52 & - \\
\hline $8-1,73$ & $=$ & $=$ & $=$ & $=$ & - & $\overline{-}$ \\
\hline $8-1,73-75$ & 1.33 & 2.55 & 7.96 & 61.1 & - & - \\
\hline $8-1,93$ & - & - & - & - & - & 332 \\
\hline $8-1,114$ & - & - & - & - & 1.52 & 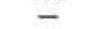 \\
\hline $8-2,50$ & - & - & - & - & - & - \\
\hline $8-2,73-75$ & 1.32 & 2.54 & 80.6 & 62.6 & - & - \\
\hline $8-2,75$ & - & - & - & - & - & 375 \\
\hline $8-2,100$ & - & - & - & - & 1.52 & - \\
\hline $8-3,39$ & $=$ & $\overline{-}$ & $=$ & $=$ & - & $\overline{-}$ \\
\hline $8-3,72$ & - & - & - & - & - & 348 \\
\hline $8-3,73-75$ & 1.34 & 2.56 & 79.6 & 60.9 & - & - \\
\hline $8-3,100$ & - & - & - & - & 1.51 & - \\
\hline
\end{tabular}


Table 1. (Continued).

\begin{tabular}{|c|c|c|c|c|c|c|}
\hline $\begin{array}{c}\text { Sample } \\
\text { (level or interval in cm) }\end{array}$ & $\underset{\left(\mathrm{g} / \mathrm{cm}^{3}\right)}{\mathrm{eb}}$ & $\underset{\left(\mathrm{g} / \mathrm{cm}^{3}\right)}{\mathrm{gg}}$ & $\stackrel{\phi}{\phi}$ & $\begin{array}{l}\text { Water } \\
\text { Content } \\
\text { (wt. \%) }\end{array}$ & $\underset{(\mathrm{km} / \mathrm{s})}{V_{\mathrm{p}}}$ & $\begin{array}{l}\text { Shear } \\
\text { Strength } \\
\left(\mathrm{g} / \mathrm{cm}^{2}\right)\end{array}$ \\
\hline $504-9-1,48$ & - & - & - & - & - & - \\
\hline $9-1,121$ & - & - & - & - & 1.52 & - \\
\hline $9-1,137$ & - & - & - & - & - & 325 \\
\hline $9-2,48$ & - & - & - & - & $=$ & - \\
\hline $9-2,72$ & $=$ & $\overline{-}$ & $\overline{-}$ & $=$ & $\overline{-}$ & 346 \\
\hline $9-2,73-75$ & 1.38 & 2.57 & 76.8 & 56.8 & - & - \\
\hline $9-2,92$ & - & - & - & - & 1.53 & - \\
\hline $9-3,49$ & - & - & - & - & - & - \\
\hline $9-3,67$ & - & $\overline{-}$ & - & - & - & 328 \\
\hline $9-3,73-75$ & 1.36 & 2.56 & 78.0 & 58.8 & - & - \\
\hline $9-3,85$ & - & - & - & - & 1.53 & - \\
\hline $10-1,76$ & - & - & - & - & - & - \\
\hline $10-1,144$ & - & - & - & - & 1.51 & - \\
\hline $10-1,147$ & - & - & - & - & - & - \\
\hline $10-2,49$ & $\overline{-}$ & $\overline{-}$ & $\overline{-}$ & $=$ & $\overline{-}$ & $\overline{-}$ \\
\hline $10-2,73-75$ & 1.29 & 2.51 & 81.8 & 64.8 & - & - \\
\hline $10-2,93$ & - & - & - & - & - & 403 \\
\hline $10-2,108$ & - & - & - & - & 1.52 & - \\
\hline $10-3,39$ & - & - & - & - & - & 309 \\
\hline $10-3,54$ & - & - & - & - & - & - \\
\hline $10-3,59$ & - & - & - & - & 1.52 & - \\
\hline $10-3,73-75$ & 1.29 & 2.49 & 81.6 & 64.5 & - & - \\
\hline $11-1$, so & - & - & - & - & - & - \\
\hline $11-1,51$ & - & - & - & - & - & - \\
\hline $11-1,70$ & $\overline{-}$ & $=$ & $\overline{-}$ & $\bar{z}$ & $1 . \overline{52}$ & $=$ \\
\hline $11-1,75-77$ & 1.56 & 2.61 & 66.0 & 43.3 & - & - \\
\hline $11-2,46$ & - & - & - & - & - & - \\
\hline $11-2,73-75$ & 1.41 & 2.58 & 75.5 & 55.0 & - & - \\
\hline $11-2,87$ & - & - & - & - & - & 386 \\
\hline $11-2,89$ & - & - & - & - & 1.53 & - \\
\hline $11-3,40$ & - & - & - & - & - & - \\
\hline $11-3,58$ & - & - & - & - & - & 302 \\
\hline $11-3,100$ & - & - & - & - & 1.51 & - \\
\hline $12-1,37$ & - & - & - & - & - & - \\
\hline $12-1,118$ & $\overline{-}$ & $\overline{-}$ & $=$ & $=$ & $=$ & 188 \\
\hline $12-1,120$ & $\bar{z}$ & $=$ & $=$ & $=$ & 1.52 & - \\
\hline $12-1,133-135$ & 1.38 & 2.56 & 76.8 & 57.0 & - & - \\
\hline $12-2,23$ & - & - & - & - & 1.52 & - \\
\hline $12-2,39$ & - & - & - & - & - & - \\
\hline $12-2,73-75$ & 1.35 & 2.52 & 78.3 & 59.5 & - & - \\
\hline $12-2,87$ & - & - & - & - & - & 386 \\
\hline $12-3,40$ & - & - & - & - & - & - \\
\hline $12-3,53$ & - & - & - & - & - & 411 \\
\hline $12-3,76$ & - & $\overline{-}$ & - & - & 1.52 & - \\
\hline $13-1,47$ & $\overline{-}$ & $\bar{z}$ & $=$ & $=$ & - & $=$ \\
\hline $13-1,62$ & - & - & - & - & - & 375 \\
\hline $13-1,35$ & - & - & - & - & 1.53 & - \\
\hline $13-1,123-125$ & 1.57 & 2.52 & 63.8 & 41.8 & - & - \\
\hline $13-2,45$ & - & - & - & - & - & - \\
\hline $13-2,61$ & - & - & - & - & - & 333 \\
\hline $13-2,73-75$ & 1.31 & 2.54 & 81.2 & 63.6 & - & - \\
\hline $13-2,82$ & - & - & - & - & 1.53 & - \\
\hline $13-3,28$ & $=$ & $\overline{-}$ & $=$ & $=$ & - & 303 \\
\hline $13-3,31$ & $=$ & $=$ & $=$ & $=$ & $=$ & - \\
\hline $13-3,42$ & - & - & - & - & 1.54 & - \\
\hline $13-3,73-75$ & 1.31 & 2.45 & 80.8 & 62.5 & - & $\overline{-}$ \\
\hline $14-1,60$ & - & - & - & - & - & - \\
\hline $14-1,68$ & - & $=$ & - & $\overline{-}$ & - & 479 \\
\hline $14-1,73-75$ & 1.28 & 2.40 & 81.2 & 64.8 & - & - \\
\hline $14-1,100$ & - & - & - & - & 1.52 & - \\
\hline $14-2,36$ & - & - & - & - & - & - \\
\hline $14-2,61$ & - & - & - & - & - & 559 \\
\hline $14-2,73-75$ & 1.25 & 2.39 & 83.8 & 69.0 & - & - \\
\hline $14-2,88$ & - & - & - & - & 1.51 & $\overline{-}$ \\
\hline $14-3,44$ & $\bar{z}$ & $=$ & $\bar{z}$ & $\bar{z}$ & - & $=$ \\
\hline $14-3,49$ & $\underline{-}$ & $=$ & $\underline{-}$ & - & - & 438 \\
\hline $14-3,73-75$ & 1.31 & 2.53 & 81.4 & 63.9 & - & - \\
\hline $14-3,88$ & - & - & - & - & 1.50 & $\overline{-}$ \\
\hline $15-1,84$ & - & - & - & - & - & 396 \\
\hline $15-1,111$ & - & - & - & - & 1.51 & - \\
\hline $15-1,123-125$ & 1.22 & 2.36 & 85.5 & 71.9 & - & - \\
\hline $15-1,133$ & - & - & - & - & - & - \\
\hline $15-2,73-75$ & $1 . \overline{22}$ & 2.37 & 85.7 & $\overline{72.2}$ & $\overline{-}$ & $=$ \\
\hline $15-2,76$ & - & - & - & - & - & 517 \\
\hline $15-2,101$ & - & - & - & $\bar{z}$ & 1.50 & - \\
\hline $15-2,122$ & $=$ & $=$ & $\overline{-}$ & $\bar{z}$ & - & $=$ \\
\hline $15-3,45$ & $=$ & - & $\underline{-}$ & $=$ & - & 504 \\
\hline $15-3,73-75$ & 1.21 & 86.0 & 86.0 & 72.8 & - & - \\
\hline $15-3,95$ & - & - & - & - & - & - \\
\hline $15-3,100$ & - & - & - & - & 1.51 & - \\
\hline $16-1,40$ & - & - & - & - & - & - \\
\hline $16-1,68$ & - & - & - & - & - & 490 \\
\hline $16-1,73-75$ & 1.26 & 2.38 & 82.5 & 67.0 & - & - \\
\hline $16-1,90$ & - & - & - & - & 1.53 & - \\
\hline $16-2,40$ & - & $=$ & $\underline{-}$ & $=$ & - & - \\
\hline $16-2,73-75$ & 1.22 & $2 . \overline{24}$ & 83.5 & 69.8 & $\bar{z}$ & $=$ \\
\hline $16-2,87$ & - & - & - & - & - & 667 \\
\hline $16-2,102$ & - & - & - & - & 1.53 & - \\
\hline $16-3,20$ & - & - & - & - & - & 508 \\
\hline $16-3,40$ & - & - & - & - & - & - \\
\hline $16-3,43$ & - & - & - & - & 1.53 & - \\
\hline
\end{tabular}


Table 1. (Continued).

\begin{tabular}{|c|c|c|c|c|c|c|}
\hline $\begin{array}{c}\text { Sample } \\
\text { (level or interval in cm) }\end{array}$ & $\underset{\left(\mathrm{g} / \mathrm{cm}^{3}\right)}{\mathrm{eb}}$ & $\left(\mathrm{g} / \mathrm{cm}^{3}\right)$ & $\begin{array}{c}\phi \\
(\%)\end{array}$ & $\begin{array}{l}\text { Water } \\
\text { Content } \\
\text { (wt.\%) }\end{array}$ & $\begin{array}{c}V_{\mathrm{p}} \\
(\mathrm{km} / \mathrm{s})\end{array}$ & $\begin{array}{l}\text { Shear } \\
\text { Strength } \\
\left(\mathrm{g} / \mathrm{cm}^{2}\right)\end{array}$ \\
\hline $504-16-3,73-75$ & 1.23 & 1.23 & 85.1 & 71.1 & - & - \\
\hline $17-1,60$ & - & - & - & - & - & - \\
\hline $17-1,61$ & - & - & - & - & - & 594 \\
\hline $17-1,73-75$ & 1.27 & 2.41 & 82.2 & 66.2 & - & - \\
\hline $17-1,89$ & - & - & - & - & 1.53 & - \\
\hline $17-2,40$ & $=$ & $=$ & $=$ & $=$ & - & $=$ \\
\hline $17-2,71$ & $=$ & $\overline{-}$ & $=$ & $=$ & - & 761 \\
\hline $17-2,130$ & - & - & $=$ & $=$ & 1.53 & - \\
\hline $17-3,40$ & - & - & $=$ & - & - & - \\
\hline $17-3,43$ & $=$ & $\overline{-}$ & $\bar{z}$ & $=$ & - & 927 \\
\hline $17-3,69$ & - & - & - & - & 1.52 & - \\
\hline $17-3,73-75$ & 1.27 & 2.34 & 81.7 & 66.2 & - & - \\
\hline $18-1,58$ & - & - & - & - & - & - \\
\hline $18-1,73-75$ & 1.24 & 2.43 & 84.4 & 69.6 & - & - \\
\hline $18-1,89$ & - & - & - & - & 1.50 & $=$ \\
\hline $\begin{array}{l}18-1,108 \\
108\end{array}$ & $\bar{z}$ & $=$ & $=$ & $\overline{-}$ & - & 534 \\
\hline $18-2,58$ & - & $=$ & $=$ & - & - & - \\
\hline $18-2,73-75$ & 1.32 & 2.27 & 76.5 & 59.5 & $=$ & - \\
\hline $18-2,18$ & - & - & - & - & $=$ & 610 \\
\hline $18-2,88$ & - & - & $=$ & - & 1.50 & - \\
\hline $18-3,45$ & - & - & - & - & - & - \\
\hline $18-3,67$ & - & - & - & - & - & 543 \\
\hline $18-3,73-75$ & 1.39 & 2.45 & 74.7 & 55.2 & - & - \\
\hline $18-3,77$ & - & - & - & - & 1.52 & - \\
\hline $19-1,40$ & - & - & $=$ & - & - & - \\
\hline $19-1,73-75$ & 1.27 & 2.38 & 82.2 & 66.6 & - & - \\
\hline $19-1,77$ & - & - & - & - & - & 543 \\
\hline $19-1,113$ & $=$ & $\bar{z}$ & $\bar{z}$ & $=$ & $1 . \overline{53}$ & - \\
\hline $19-2,40$ & $=$ & - & - & - & - & - \\
\hline $19-2,61$ & - & - & - & - & - & 543 \\
\hline $19-2,73-75$ & 1.35 & 2.61 & 79.6 & 60.5 & - & - \\
\hline $19-2,99$ & - & - & - & - & 1.51 & - \\
\hline $20-1,70$ & - & - & - & - & 1.51 & - \\
\hline $20-1,73-75$ & 1.33 & 2.62 & 80.9 & 62.4 & - & - \\
\hline $20-1,95$ & - & - & - & - & - & 549 \\
\hline $20-2,73-75$ & 1.35 & 2.55 & 78.9 & 60.0 & - & - \\
\hline $20-2,84$ & - & - & - & - & - & 520 \\
\hline $20-2,115$ & - & - & - & - & 1.51 & - \\
\hline $20-3,6 ?$ & $=$ & $\bar{z}$ & $=$ & $=$ & - & 572 \\
\hline $20-2,73-75$ & 1.35 & 2.55 & $\overline{78.6}$ & 59.6 & $\bar{z}$ & - \\
\hline $20-3,95$ & - & - & - & - & 1.52 & $\overline{-}$ \\
\hline $21-1,63$ & $=$ & $=$ & $=$ & $=$ & 1.22 & $=$ \\
\hline $21-1,72$ & $\overline{-}$ & - & - & - & - & 344 \\
\hline $21-1,136$ & $=$ & - & - & - & 1.51 & - \\
\hline $21-2,35$ & - & - & - & - & - & - \\
\hline $21-2,10$ & - & - & - & - & - & 251 \\
\hline $21-2,105$ & - & - & - & - & 1.52 & - \\
\hline $21-3,45$ & - & - & - & - & - & - \\
\hline $21-3,61$ & - & - & - & - & - & 695 \\
\hline $21-3,73-75$ & $1 . \overline{49}$ & 2.59 & $\overline{70.6}$ & 48.6 & $\overline{-}$ & - \\
\hline $21-3,99$ & - & - & - & - & 1.52 & - \\
\hline $22-1,73-75$ & 1.23 & 2.37 & 84.6 & $\overline{70.5}$ & - & $\overline{-}$ \\
\hline $22-1,112$ & - & - & - & - & - & - \\
\hline $22-1,121$ & $\overline{-}$ & $=$ & $=$ & $=$ & $=$ & 397 \\
\hline $22-1,136$ & - & - & - & - & 1.52 & - \\
\hline $22-2,39$ & - & - & - & - & - & - \\
\hline $22-2,73-75$ & 1.29 & 2.50 & 818 & 64.8 & - & - \\
\hline $22-2,76$ & - & - & - & - & - & 526 \\
\hline $22-2,93$ & - & - & - & - & 1.50 & - \\
\hline $23-1,73$ & - & - & - & - & - & 333 \\
\hline $23-1,73-75$ & 1.29 & 2.68 & 83.9 & $\overline{66.6}$ & $=$ & - \\
\hline $23-1,117$ & 1.29 & 2.08 & $\begin{array}{l}83.9 \\
-\end{array}$ & ${ }_{-}^{\infty} .6$ & $\bar{z}$ & $=$ \\
\hline $23-1,124$ & $\overline{-}$ & $\overline{-}$ & $=$ & $\overline{-}$ & 1.52 & $\overline{-}$ \\
\hline $24-1,73-75$ & 1.36 & 2.58 & 78.2 & 58.7 & - & $\bar{z}$ \\
\hline $24-1,92$ & - & - & - & - & $\overline{-}$ & 491 \\
\hline $24-1,100$ & $=$ & $=$ & $\overline{-}$ & $=$ & $\overline{-}$ & - \\
\hline $24-1,117$ & - & - & - & - & 1.52 & - \\
\hline $24-2,29$ & - & - & - & - & - & - \\
\hline $24-2,41$ & - & - & - & - & 1.52 & - \\
\hline $24-2,60$ & $\overline{-}$ & - & $\overline{-}$ & - & - & 444 \\
\hline $24-2,73-75$ & 1.35 & 2.63 & 79.6 & 60.3 & - & - \\
\hline $25-1,73-75$ & 1.37 & 2.64 & 78.5 & 58.6 & $=$ & - \\
\hline $25-1,91$ & - & -2.04 & - & 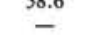 & $=$ & $\overline{689}$ \\
\hline $25-1,113$ & $\overline{-}$ & $=$ & $=$ & $=$ & 1.52 & - \\
\hline $25-1,116$ & $=$ & $=$ & $\bar{z}$ & $=$ & -2.52 & $=$ \\
\hline $25-2,57$ & - & - & - & - & - & - \\
\hline $25-2,61$ & - & - & - & - & - & 870 \\
\hline $25-2,73-75$ & 1.32 & 2.55 & 80.5 & 62.4 & - & - \\
\hline $25-2,120$ & - & - & - & - & 1.52 & - \\
\hline $25-3,35$ & - & - & - & - & - & - \\
\hline $25-3,55$ & - & - & - & $=$ & $=$ & 537 \\
\hline $25-3,65$ & - & - & - & - & 1.50 & - \\
\hline $26-1,59$ & $=$ & $=$ & - & $\overline{-}$ & - & $\overline{-}$ \\
\hline $26-1,66$ & $\bar{z}$ & $=$ & $\bar{z}$ & $\overline{-}$ & $=$ & $\overline{543}$ \\
\hline $26-1,73-75$ & 1.34 & 2.56 & 79.4 & 60.7 & - & - \\
\hline $26-1,107$ & - & - & - & - & 1.51 & $=$ \\
\hline $26-2,41$ & $\bar{z}$ & $\bar{z}$ & $\bar{z}$ & $\bar{z}$ & 1.21 & $\bar{z}$ \\
\hline $26-2,50$ & - & - & - & - & - & 759 \\
\hline $26-2,73-75$ & 1.31 & 2.49 & 80.2 & 62.5 & - & - \\
\hline $26-2,112$ & - & - & - & - & 1.52 & - \\
\hline $26-3,33$ & - & - & - & - & - & - \\
\hline
\end{tabular}


Table 1. (Continued).

\begin{tabular}{|c|c|c|c|c|c|c|c|}
\hline $\begin{array}{c}\text { Sample } \\
\text { (level or interval in } \mathrm{cm} \text { ) }\end{array}$ & $\underset{\left(\mathrm{g} / \mathrm{cm}^{3}\right)}{\mathrm{eb}}$ & $\left(\mathrm{g} / \mathrm{cm}^{\mathrm{eg}}\right)$ & $\stackrel{\phi}{(\%)}$ & $\begin{array}{l}\text { Water } \\
\text { Content } \\
\text { (wt.\%) }\end{array}$ & $\underset{(\mathrm{km} / \mathrm{s})}{V_{\mathrm{p}}}$ & $\begin{array}{l}\text { Shear } \\
\text { Strength } \\
\left(\mathrm{g} / \mathrm{cm}^{2}\right)\end{array}$ & $\begin{array}{c}\text { Penetrability } \\
(\mathrm{mm})\end{array}$ \\
\hline $504-26-3,57$ & - & - & - & - & - & 718 & 4.6 \\
\hline $26-3,68$ & - & - & - & - & 1.51 & - & - \\
\hline $26-3,73-75$ & 1.35 & 2.57 & 79.1 & 60.1 & - & - & - \\
\hline $27-1,44$ & - & - & - & - & - & - & - \\
\hline $27-1,58$ & - & - & - & - & - & 701 & 4.4 \\
\hline $27-1,73-75$ & 1.34 & 2.55 & 79.1 & 60.2 & - & - & - \\
\hline $27-1,104$ & - & - & - & - & 1.52 & - & - \\
\hline $27-2,55$ & - & - & - & - & - & - & - \\
\hline $27-2,64$ & - & - & - & - & - & 818 & 4.9 \\
\hline $27-2,73-75$ & 1.32 & 2.54 & 80.6 & 62.6 & - & - & - \\
\hline $27-2,96$ & - & - & - & - & 1.51 & - & - \\
\hline $27-3,30$ & - & - & - & - & - & - & - \\
\hline $27-3,33$ & - & - & - & - & - & 695 & 4.3 \\
\hline $27-3,47$ & - & - & - & - & 1.52 & - & - \\
\hline $27-3,73-75$ & 1.35 & 2.57 & 79.2 & 60.3 & - & - & - \\
\hline $28-1,71$ & - & - & - & - & - & - & - \\
\hline $28-1,127$ & - & - & - & - & 1.51 & - & - \\
\hline $28-1,134$ & - & - & - & - & - & 526 & 5.7 \\
\hline $28-1,137-139$ & 1.33 & 2.57 & 80.5 & 62.2 & - & - & - \\
\hline $28-2,33$ & - & - & - & - & - & 654 & 4.9 \\
\hline $28-2,35$ & - & - & - & - & - & - & - \\
\hline $28-2,47$ & - & - & $=$ & - & 1.52 & - & - \\
\hline $28-2,73-75$ & 1.34 & 2.60 & 80.0 & 61.3 & - & $=$ & $=$ \\
\hline $29-1,73-75$ & 1.33 & 2.52 & 79.7 & 61.5 & - & - & - \\
\hline $29-1,78$ & - & - & - & - & - & 701 & 4.8 \\
\hline $29-1,104$ & - & - & - & - & 1.52 & $\overline{-1}$ & - \\
\hline $29-2,74$ & - & - & - & - & - & 747 & 8.1 \\
\hline $29-2,100$ & - & - & - & - & 1.53 & - & - \\
\hline $29-2,123-125$ & 1.36 & 2.58 & 78.3 & 58.9 & - & - & $\overline{-}$ \\
\hline $29-3,44$ & - & - & - & - & - & 742 & 4.9 \\
\hline $29-3,68$ & - & - & - & - & 1.51 & - & - \\
\hline $29-3,73-75$ & 1.35 & 2.56 & 78.6 & 59.5 & - & - & - \\
\hline $30-1,39$ & - & - & - & - & - & - & - \\
\hline $30-1,47$ & - & - & - & 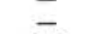 & - & 1133 & 4.2 \\
\hline $30-1,73-75$ & 1.29 & 2.20 & 77.3 & 61.3 & - & - & - \\
\hline $30-1,82$ & - & - & - & - & 1.51 & - & - \\
\hline $30-2,36$ & - & - & - & - & - & - & - \\
\hline $30-2,73-75$ & 1.33 & 2.26 & 75.0 & 57.6 & - & - & - \\
\hline $30-2,91$ & - & - & - & - & - & 1051 & 4.6 \\
\hline $30-2,101$ & - & - & - & - & 1.50 & - & - \\
\hline $30-3,17$ & - & - & - & $=$ & - & 1051 & 4.6 \\
\hline $30-3,22$ & - & - & - & - & - & - & - \\
\hline $30-3,42$ & - & - & - & - & 1.49 & - & - \\
\hline $30-3,43-45$ & 1.34 & 2.25 & 74.4 & 56.7 & - & - & - \\
\hline $31-1,73-75$ & 1.37 & 2.33 & 73.3 & 54.7 & - & - & - \\
\hline $31-1,82$ & - & - & - & - & - & - & - \\
\hline $31-1,94$ & - & - & - & - & - & 1121 & 4.4 \\
\hline $31-1,114$ & - & - & - & - & 1.48 & - & - \\
\hline $31-2,35$ & - & - & - & - & - & - & - \\
\hline $31-2,73-75$ & 1.40 & 2.37 & 72.0 & 52.7 & - & - & - \\
\hline $31-2,80$ & - & - & - & - & 1.50 & - & $=$ \\
\hline $32-1,43$ & - & - & - & - & - & - & - \\
\hline $32-1,73-75$ & 1.38 & 2.34 & 73.1 & 54.4 & - & - & - \\
\hline $32-1,104$ & - & - & - & - & 1.50 & - & - \\
\hline $32-1,49$ & - & - & - & - & - & 1168 & 4.2 \\
\hline $32-2,49$ & - & - & - & - & - & - & - \\
\hline $32-2,60$ & - & - & - & - & 1.51 & - & - \\
\hline $32-2,73-75$ & 1.37 & 2.46 & 75.9 & 56.8 & - & - & - \\
\hline $32-3,23-25$ & 1.46 & 2.39 & 67.9 & 47.6 & - & - & - \\
\hline $32-3,27$ & - & - & - & - & - & - & - \\
\hline $32-3,33$ & $=$ & $=$ & $=$ & $\overline{-}$ & 1.53 & $=$ & $=$ \\
\hline $33-1,45$ & - & - & - & - & - & - & - \\
\hline $33-1,73-75$ & 1.43 & 2.37 & 69.8 & 49.9 & $\overline{-}$ & - & - \\
\hline $33-1,91$ & - & - & - & - & - & 700 & 5.4 \\
\hline $33-1,98$ & - & - & - & - & 1.55 & - & - \\
\hline $33-2,16$ & - & - & - & - & - & - & - \\
\hline $33-2,20$ & - & - & - & - & 1.54 & - & - \\
\hline $34-1,68$ & - & - & - & - & - & - & - \\
\hline $34-1,73-75$ & 1.29 & 2.20 & 77.4 & 61.4 & - & - & - \\
\hline $34-1,101$ & - & - & - & - & $\overline{-}$ & 724 & 3.9 \\
\hline $34-1,104$ & - & - & - & - & 1.54 & - & - \\
\hline $34-1,110$ & - & - & - & $\overline{-}$ & - & - & $\overline{-}$ \\
\hline $34-3,50$ & $\overline{-}$ & $\overline{-}$ & $=$ & $=$ & $\overline{-}$ & $\overline{-}$ & $=$ \\
\hline $34-2,73-75$ & 1.39 & 2.35 & 72.8 & 53.8 & - & - & - \\
\hline $34-2,99$ & - & - & - & - & 1.53 & - & - \\
\hline $34-3,18$ & - & - & - & - & 1.52 & - & - \\
\hline $35-1,31$ & - & - & - & - & - & - & - \\
\hline $35-1,73-75$ & 1.32 & 2.23 & 75.5 & 58.6 & - & - & - \\
\hline $35-1,107$ & - & - & - & - & 1.53 & - & - \\
\hline $35-1,116$ & - & - & - & - & - & 1203 & 4.1 \\
\hline $35-2,38$ & - & - & - & - & - & - & - \\
\hline $35-2,73-75$ & 1.37 & 2.29 & 72.9 & 54.5 & - & - & - \\
\hline $35-2,103$ & - & - & - & - & 1.54 & - & - \\
\hline $\begin{array}{l}35-3,35 \\
35-3,56\end{array}$ & $\bar{z}$ & $\overline{-}$ & $=$ & $=$ & $\overline{152}$ & - & $=$ \\
\hline $\begin{array}{l}35-3,56 \\
36-1,41\end{array}$ & $\overline{-}$ & $=$ & $=$ & $\bar{z}$ & 1.52 & $=$ & $\bar{z}$ \\
\hline $\begin{array}{l}36-1,41 \\
36-1,73-75\end{array}$ & $1 . \overline{47}$ & $2 . \overline{42}$ & 57.7 & $\overline{47.1}$ & $=$ & $\overline{-}$ & $\bar{z}$ \\
\hline $36-1,75$ & - & - & - & - & - & 1670 & 4.2 \\
\hline $36-1,98$ & - & - & - & - & 1.53 & - & - \\
\hline $36-2,40$ & - & - & - & - & - & - & - \\
\hline $36-2,73-75$ & 1.49 & 2.41 & 66.1 & 45.4 & - & - & - \\
\hline
\end{tabular}


Table 1. (Continued).

\begin{tabular}{|c|c|c|c|c|c|c|}
\hline $\begin{array}{c}\text { Sample } \\
\text { (level or interval in cm) }\end{array}$ & $\underset{\left(\mathrm{g} / \mathrm{cm}^{3}\right)}{\mathrm{eb}}$ & $\left(\mathrm{g} / \mathrm{cm}^{3}\right)$ & $\begin{array}{c}\phi \\
(\%)\end{array}$ & $\begin{array}{l}\text { Water } \\
\text { Content } \\
\text { (wt.\%) }\end{array}$ & $\begin{array}{c}V_{p} \\
(\mathrm{~km} / \mathrm{s})\end{array}$ & $\begin{array}{l}\text { Shear } \\
\text { Strength } \\
\left(\mathrm{g} / \mathrm{cm}^{2}\right)\end{array}$ \\
\hline $504-36-2,88$ & - & - & - & - & 1.53 & - \\
\hline $37-1,33$ & - & - & - & - & - & - \\
\hline $37-1,96$ & - & - & - & - & - & 999 \\
\hline $37-1,125$ & - & - & - & - & 1.54 & - \\
\hline $37-2,34$ & - & - & - & - & - & - \\
\hline $37-2,63$ & - & - & - & - & - & 1361 \\
\hline $37-2,94$ & - & - & - & - & 1.53 & $\overline{-1}$ \\
\hline $38-1,73$ & - & - & - & - & - & 1192 \\
\hline $38-1,102$ & - & - & - & - & 1.54 & - \\
\hline $38-1,129$ & - & - & - & - & - & - \\
\hline $38-2,79$ & - & - & - & - & - & 1250 \\
\hline $38-2,97$ & - & - & - & - & 1.54 & - \\
\hline $38-2,127$ & - & - & - & - & - & - \\
\hline $38-3,48$ & - & - & - & - & - & 829 \\
\hline $38-3,58$ & - & - & - & - & - & - \\
\hline $38-3,61$ & - & - & - & - & 1.52 & - \\
\hline $40-1,59$ & - & - & - & - & - & - \\
\hline $40-1,65$ & - & - & - & - & - & 625 \\
\hline $40-1,83$ & - & - & - & - & 1.53 & - \\
\hline $40-2,27$ & - & - & - & - & - & - \\
\hline $40-2,34$ & - & - & - & - & - & 438 \\
\hline $40-2,50$ & - & - & - & - & 1.53 & - \\
\hline $40-1,83$ & - & - & - & - & - & - \\
\hline $40-1,95$ & - & - & - & - & 1.54 & - \\
\hline $40-1,107$ & - & - & - & - & - & 356 \\
\hline $40-1,136$ & - & - & - & - & 1.658 & - \\
\hline $41-2,35$ & - & - & - & - & - & - \\
\hline $41-2,41$ & - & - & - & - & - & 754 \\
\hline $41-2,70$ & - & - & - & - & 1.53 & - \\
\hline $41-2,73-75$ & 1.53 & 2.47 & 64.8 & 43.3 & - & - \\
\hline $41-3,53$ & - & - & - & - & - & - \\
\hline $41-3,72$ & - & - & - & - & - & 800 \\
\hline $41-3,73-75$ & 1.51 & 2.43 & 65.8 & 44.7 & - & - \\
\hline $41-3,109$ & - & - & - & - & 1.54 & - \\
\hline $42-1,61$ & - & - & - & - & - & 537 \\
\hline $42-1,63$ & - & - & - & - & - & - \\
\hline $42-1,73-75$ & 1.56 & 2.46 & 63.0 & 41.5 & - & - \\
\hline $42-1,115$ & - & - & - & - & 1.52 & - \\
\hline $42-2,30$ & - & - & - & - & - & 426 \\
\hline $42-2,32$ & - & - & - & - & - & - \\
\hline $42-2,68$ & - & - & $=$ & - & 1.53 & - \\
\hline $43-1,54$ & - & - & - & - & - & - \\
\hline $43-1,62$ & - & - & - & - & - & 642 \\
\hline $43-1,73-75$ & 1.54 & 2.46 & 63.9 & 42.4 & - & - \\
\hline $43-1,105$ & - & - & - & - & 1.52 & - \\
\hline $43-2,45$ & - & - & - & - & - & - \\
\hline $43-2,70$ & - & - & - & - & - & 923 \\
\hline $43-2,73-75$ & 1.55 & 2.48 & 63.6 & 41.9 & - & - \\
\hline $43-2,112$ & - & - & - & - & 1.52 & - \\
\hline $43-3,45$ & - & - & - & - & - & - \\
\hline $43-3,73$ & - & - & - & - & - & 631 \\
\hline $43-3,73-75$ & 1.46 & 2.40 & 68.2 & 47.7 & - & - \\
\hline $43-3,93$ & - & - & - & - & 1.52 & - \\
\hline $44-1,63$ & - & - & - & - & - & - \\
\hline $44-1,73-75$ & 1.47 & 2.42 & 67.8 & 47.2 & - & - \\
\hline $44-1,79$ & - & - & - & - & - & 543 \\
\hline $44-1,91$ & - & - & $=$ & - & 1.53 & - \\
\hline $44-2,11$ & $=$ & - & $=$ & - & - & 368 \\
\hline $44-2,26$ & - & - & - & - & - & - \\
\hline $44-2,54$ & - & $\overline{-}$ & $=$ & $=$ & 1.54 & $\overline{-}$ \\
\hline $45-1,49$ & - & - & - & - & - & - \\
\hline $45-1,73-75$ & 1.51 & 2.46 & 66.4 & 45.2 & - & - \\
\hline $45-1,98$ & - & - & - & - & - & 456 \\
\hline $45-1,110$ & - & - & - & - & 1.53 & - \\
\hline $45-2,24$ & - & - & - & - & - & - \\
\hline $45-2,37$ & - & - & - & - & - & 409 \\
\hline $45-2,44$ & - & - & - & - & 1.52 & - \\
\hline $46-1,66$ & - & - & $=$ & $\overline{-}$ & - & 672 \\
\hline $46-1,73-75$ & 1.39 & 2.33 & 72.1 & 53.3 & - & - \\
\hline $46-1,112$ & - & - & - & - & - & - \\
\hline $46-1,133$ & - & - & - & - & 1.52 & - \\
\hline $47-1,73-75$ & 1.42 & 2.34 & 69.8 & 50.2 & - & - \\
\hline $47-1,74$ & - & - & - & - & - & 642 \\
\hline $47-1,104$ & - & - & - & - & 1.54 & - \\
\hline $47-1,109$ & - & $\overline{-}$ & $=$ & $\overline{-}$ & - & - \\
\hline $48-1,65$ & - & - & - & - & - & 561 \\
\hline $48-1,65$ & - & - & - & - & - & - \\
\hline $48-1,73-75$ & 1.52 & 2.44 & 64.8 & 43.6 & $\overline{-}$ & $=$ \\
\hline $48-1,89$ & - & - & - & - & 1.54 & - \\
\hline $49-1,63$ & - & - & - & - & - & 432 \\
\hline $49-1,73-75$ & 1.45 & 2.38 & $\overline{68.3}$ & 48.1 & $=$ & - \\
\hline $49-1,84$ & - & - & - & - & - & - \\
\hline $49-1,121$ & - & - & - & - & 1.55 & - \\
\hline $50-1,52$ & - & - & - & - & - & - \\
\hline $50-1,73-75$ & 1.41 & 2.30 & 70.0 & 51.0 & $\overline{-}$ & $\overline{-}$ \\
\hline $50-1,87$ & ${ }_{-1.1}^{-12}$ & - & - & - & $\overline{-}$ & 525 \\
\hline $50-1,115$ & - & - & - & - & 1.58 & - \\
\hline $51-1,46$ & $=$ & - & $=$ & $=$ & - & $=$ \\
\hline $51-1,69$ & - & - & - & - & - & 584 \\
\hline $51-1,73-75$ & 1.50 & 2.39 & 65.0 & 44.3 & - & - \\
\hline $51-1,80$ & - & - & - & - & 1.55 & - \\
\hline
\end{tabular}


Table 1. (Continued).

\begin{tabular}{|c|c|c|c|c|c|c|}
\hline $\begin{array}{c}\text { Sample } \\
\text { (level or interval in } \mathrm{cm} \text { ) }\end{array}$ & $\underset{\left(\mathrm{g} / \mathrm{cm}^{3}\right)}{\mathrm{eb}}$ & $\underset{\left(\mathrm{g} / \mathrm{cm}^{3}\right)}{\mathrm{g}^{3}}$ & $\begin{array}{c}\phi \\
(\%)\end{array}$ & $\begin{array}{l}\text { Water } \\
\text { Content } \\
\text { (wt. \%) }\end{array}$ & $(\mathrm{km} / \mathrm{s})$ & $\begin{array}{l}\text { Shear } \\
\text { Strength } \\
\left(\mathrm{g} / \mathrm{cm}^{2}\right)\end{array}$ \\
\hline $504-51-1,127$ & - & - & - & - & - & - \\
\hline $52-1,49$ & - & - & - & - & - & 584 \\
\hline $52-1,59$ & - & - & - & - & 1.55 & - \\
\hline $52-1,80$ & - & - & - & - & - & - \\
\hline $53-1,30-32$ & 143 & 2.35 & 69.1 & 49.4 & - & - \\
\hline $53-1,46$ & - & - & - & - & - & - \\
\hline $505-2-2,54$ & - & - & - & - & - & \\
\hline $2-2,73-75$ & 1.24 & 2.64 & 86.9 & 72.1 & - & \\
\hline $2-2,106$ & - & - & - & - & 1.51 & \\
\hline $2-3,73-75$ & 1.25 & 2.54 & 84.9 & 69.5 & - & \\
\hline $2-3,103$ & - & - & - & - & 1.50 & \\
\hline $2-4,86-88$ & 1.25 & 2.64 & 86.2 & 70.8 & - & \\
\hline $2-4,92$ & - & - & - & - & - & \\
\hline $2-4,97$ & - & - & - & - & 1.50 & \\
\hline $2-5,43$ & - & - & - & - & 1.52 & \\
\hline $2-6,68$ & - & - & - & - & - & \\
\hline $3-2,11$ & - & - & - & - & 1.50 & \\
\hline $3-2,14-16$ & 1.24 & 2.73 & 87.4 & 72.2 & - & \\
\hline $3-2,70$ & - & - & - & - & - & \\
\hline $3-3,73-75$ & 1.23 & 2.71 & 88.1 & 73.6 & - & \\
\hline $3-3,121$ & - & - & - & - & 1.50 & \\
\hline $3-5,125$ & - & - & - & - & 1.52 & \\
\hline $3-5,130-132$ & 1.23 & 2.64 & 86.7 & 72.5 & - & \\
\hline $4-2,75$ & - & - & - & - & - & \\
\hline $4-2,101-103$ & 1.32 & 2.72 & 82.4 & 63.9 & - & \\
\hline $4-2,122$ & - & - & - & - & 1.53 & \\
\hline $4-3,95-97$ & 1.31 & 2.65 & 82.4 & 64.4 & - & \\
\hline $4-3,102$ & - & - & - & - & 1.51 & \\
\hline $4-4,48$ & - & - & - & - & - & \\
\hline $4-4,71-73$ & 1.28 & 2.74 & 85.1 & 68.1 & - & \\
\hline $4-4,100$ & - & - & - & - & 1.50 & \\
\hline $4-5,84-86$ & 1.29 & 2.65 & 83.9 & 66.9 & - & \\
\hline $4-6,46$ & - & - & - & - & - & \\
\hline $4-6,90$ & - & - & - & - & 1.51 & \\
\hline $4-7,73-75$ & 1.34 & 2.71 & 81.3 & 62.2 & - & \\
\hline $5-1,73-75$ & 1.29 & 2.78 & 84.9 & 67.5 & - & \\
\hline $5-2,43$ & - & - & - & - & - & \\
\hline $5-2,73-75$ & 1.25 & 2.73 & 86.7 & 70.9 & - & \\
\hline $5-2,124$ & - & - & - & - & 1.51 & \\
\hline $5-3,73-75$ & 1.31 & 2.73 & 83.1 & 64.9 & - & \\
\hline $5-4,40$ & - & - & - & - & - & \\
\hline $5-4,73-75$ & 1.29 & 2.64 & 83.3 & 65.9 & - & \\
\hline $5-4,117$ & - & - & - & - & 1.52 & \\
\hline $5-5,73-75$ & 1.27 & 2.62 & 84.4 & 68.0 & - & \\
\hline $5-6,41$ & - & - & - & - & - & \\
\hline $5-6,73-75$ & 1.25 & 2.64 & 86.0 & 70.4 & - & \\
\hline $5-6,105$ & - & - & - & - & 1.52 & \\
\hline $6-1,53-55$ & 1.25 & 2.55 & 85.0 & 69.4 & - & \\
\hline $6-2,44$ & - & - & - & - & - & \\
\hline $6-2,73-75$ & 1.27 & 2.63 & 84.4 & 67.9 & - & \\
\hline $6-3,73-75$ & 1.25 & 2.65 & 86.2 & 70.8 & - & \\
\hline $6-4,45$ & - & - & - & - & - & \\
\hline $6-4,73-75$ & 1.28 & 2.64 & 83.9 & 66.9 & - & \\
\hline $6-4,105$ & - & - & - & - & 1.49 & \\
\hline $6-5,73-75$ & 1.33 & 2.65 & 81.5 & 63.0 & - & \\
\hline $6-6,44$ & - & - & - & - & - & \\
\hline $6-6,73-75$ & 1.31 & 2.62 & 82.3 & 64.5 & - & \\
\hline $6-6,99$ & - & - & - & - & 1.50 & \\
\hline $6-7,73-75$ & 1.25 & 2.56 & 85.2 & 69.7 & - & \\
\hline $7-1,73-75$ & 1.26 & 2.55 & 84.7 & 69.0 & - & \\
\hline $7-2,39$ & - & - & - & - & - & \\
\hline $7-2,73-75$ & 1.27 & 2.59 & 84.0 & 67.5 & - & \\
\hline $7-2,84$ & - & - & - & - & 1.52 & \\
\hline $7-3,73-75$ & 1.30 & 2.58 & 82.0 & 64.5 & - & \\
\hline $7-4,44$ & - & - & - & - & - & \\
\hline $7-4,73-75$ & 1.31 & 2.64 & 82.3 & 64.4 & - & \\
\hline $7-4,102$ & - & - & - & - & 1.50 & \\
\hline $7-5,73-75$ & 1.34 & 2.52 & 79.2 & 60.7 & - & \\
\hline $7-6,28$ & - & - & - & - & 1.52 & \\
\hline $7-6,36$ & - & - & - & - & - & \\
\hline $7-6,73-75$ & 1.32 & 2.59 & 80.9 & 62.7 & - & \\
\hline $9-1,100-102$ & 1.35 & 2.61 & 79.7 & 60.7 & - & \\
\hline $9-2,47$ & - & - & - & - & - & \\
\hline $9-2,73-75$ & 1.37 & 2.94 & 82.0 & 61.3 & - & \\
\hline $9-2,94$ & - & - & - & - & 1.51 & \\
\hline $9-3,73-75$ & 1.29 & 2.59 & 82.8 & 65.6 & - & \\
\hline $9-4,14$ & - & - & - & - & - & \\
\hline $10-1,73-75$ & 1.32 & 2.66 & 82.1 & 63.7 & - & \\
\hline $10-1,126$ & - & - & - & - & 1.51 & \\
\hline $10-2,42$ & - & - & - & - & - & \\
\hline $10-2,73-75$ & 1.26 & 2.52 & 84.0 & 68.2 & - & \\
\hline $10-2,119$ & - & - & - & - & 1.51 & \\
\hline $10-3,73-75$ & 1.34 & 2.60 & 79.8 & 60.8 & - & \\
\hline $10-3,110$ & - & - & - & - & 1.52 & \\
\hline $10-4,46$ & - & - & - & - & - & \\
\hline $10-4,73-75$ & 1.37 & 2.66 & 78.8 & 58.8 & - & \\
\hline $10-4,125$ & - & - & - & - & 1.51 & \\
\hline $10-5,73-75$ & 1.31 & 2.55 & 81.6 & 64.0 & - & \\
\hline $11-1,100-102$ & 1.29 & 2.54 & 82.2 & 65.1 & - & \\
\hline $11-1,135$ & - & - & - & - & 1.52 & \\
\hline $11-2.73-75$ & 1.26 & 2.50 & 84.3 & 68.8 & - & \\
\hline
\end{tabular}


Table 1. (Continued).

\begin{tabular}{|c|c|c|c|c|c|c|}
\hline $\begin{array}{c}\text { Sample } \\
\text { (level or interval in } \mathrm{cm} \text { ) }\end{array}$ & $\stackrel{\substack{\mathrm{eb} \\
\left(\mathrm{g} / \mathrm{cm}^{3}\right)}}{2}$ & $\underset{\left(\mathrm{g} / \mathrm{cm}^{3}\right)}{\mathrm{gg}}$ & $\begin{array}{l}\phi \\
(\%)\end{array}$ & $\begin{array}{l}\text { Water } \\
\text { Content } \\
\text { (wt. \%) }\end{array}$ & $\underset{(\mathrm{km} / \mathrm{s})}{V_{\mathrm{p}}}$ & $\begin{array}{l}\text { Shear } \\
\text { Strength } \\
\left(\mathrm{g} / \mathrm{cm}^{2}\right)\end{array}$ \\
\hline $505-11-2,82$ & - & - & - & - & - & \\
\hline $11-2,130$ & - & - & - & - & 1.52 & \\
\hline $11-3,73-75$ & 1.24 & 2.47 & 84.8 & 69.9 & - & \\
\hline $11-3,127$ & - & - & - & - & 1.52 & \\
\hline $11-4,64$ & - & - & - & - & - & \\
\hline $11-4,73-75$ & 1.24 & 2.55 & 85.9 & 71.0 & - & \\
\hline $11-4,124$ & - & - & - & - & 1.50 & \\
\hline $11-5,73-75$ & 1.23 & 2.49 & 86.0 & 71.6 & - & \\
\hline $11-5,95$ & - & - & - & - & 1.52 & \\
\hline $11-6,18$ & $=$ & - & - & - & 1.52 & \\
\hline $11-6,73$ & - & - & - & - & 1.52 & \\
\hline $11-6,73-75$ & 1.21 & 2.46 & 86.8 & 73.2 & - & \\
\hline $12-1,114$ & - & - & - & - & 1.51 & \\
\hline $12-2,37-39$ & 1.26 & 2.52 & 84.2 & 68.3 & - & \\
\hline $12-2,39$ & - & - & - & - & 1.51 & \\
\hline $12-2,53$ & - & - & - & - & - & \\
\hline $12-3,73-75$ & 1.24 & 2.38 & 84.1 & 69.4 & $\overline{-}$ & \\
\hline $12-3,118$ & - & - & - & - & 1.53 & \\
\hline $12-4,36$ & $=$ & $=$ & $=$ & $=$ & - & \\
\hline $12-4,71$ & - & - & - & - & 1.52 & \\
\hline $12-4,73-75$ & 1.23 & 2.47 & 85.8 & 71.6 & - & \\
\hline $13-1,73-75$ & 1.24 & 2.40 & 84.4 & 69.9 & - & \\
\hline $13-1,135$ & - & - & - & - & 1.53 & \\
\hline $13-2,134$ & - & - & - & - & - & \\
\hline $13-3,73-75$ & 1.26 & 2.57 & 84.5 & 68.5 & - & \\
\hline $13-3,130$ & -20 & - & - & - & 1.52 & \\
\hline $13-4,48$ & $=$ & $=$ & $\bar{z}$ & $=$ & -1.02 & \\
\hline $13-4,73-75$ & 1.26 & 2.59 & 85.1 & 69.3 & - & \\
\hline $13-4,88$ & - & - & - & - & 1.52 & \\
\hline $13-5,73-75$ & 1.33 & 2.53 & 79.5 & 61.0 & - & \\
\hline $13-5,115$ & - & - & - & - & 1.51 & \\
\hline $13-6,54$ & - & - & - & - & - & \\
\hline $13-6,73-75$ & 1.31 & 2.54 & 80.8 & 63.1 & - & \\
\hline $13-6,129$ & - & - & - & - & 1.53 & \\
\hline $14-2,54$ & $=$ & $=$ & - & $\overline{-}$ & - & \\
\hline $14-2,73-75$ & 1.32 & 2.50 & 80.2 & 62.5 & $=$ & \\
\hline $14-2,75$ & - & - & - & - & 1.52 & \\
\hline $14-3,73-75$ & 1.31 & 2.55 & 81.0 & 63.1 & - & \\
\hline $14-3,129$ & - & - & - & - & 1.50 & \\
\hline $14-4,52$ & - & - & - & - & - & \\
\hline $14-4,73-75$ & 1.32 & 2.57 & 80.9 & 62.8 & - & \\
\hline $14-4,110$ & - & - & - & - & 1.52 & \\
\hline $14-5,73-75$ & 1.37 & 2.58 & 77.6 & 58.0 & - & \\
\hline $14-5,92$ & - & - & - & - & 1.52 & \\
\hline $15-2,50$ & $=$ & $=$ & $\bar{z}$ & $\overline{-}$ & - & \\
\hline $15-2,123-125$ & 1.35 & 2.59 & 79.0 & 59.7 & - & \\
\hline $15-2,132$ & - & - & - & - & 1.51 & \\
\hline $15-3,73-75$ & 1.31 & 2.56 & 81.5 & 63.7 & - & \\
\hline $15-3,139$ & - & - & - & - & 1.52 & \\
\hline $15-4,42$ & - & - & - & - & - & \\
\hline $15-4,73-75$ & 1.38 & 2.62 & 77.5 & 57.4 & - & \\
\hline $15-4,132$ & - & - & - & - & 1.52 & \\
\hline $15-5,73-75$ & 1.34 & 2.51 & 78.7 & 60.2 & - & \\
\hline $15-5,131$ & - & - & - & - & 1.52 & \\
\hline $15-6,26-28$ & 1.30 & 2.51 & 81.5 & 64.2 & - & \\
\hline $15-6,47$ & - & - & - & - & 1.52 & \\
\hline $17-1,103$ & - & $=$ & $\overline{-}$ & $=$ & 1.50 & \\
\hline $17-2,45$ & - & - & - & - & - & \\
\hline $17-2,73-75$ & 1.35 & 2.61 & 76.7 & 60.6 & - & \\
\hline $17-2,131$ & - & - & - & - & 1.51 & \\
\hline $17-3,73-75$ & 1.38 & 2.58 & 77.4 & 57.7 & - & \\
\hline $17-3,136$ & - & - & - & - & 1.50 & \\
\hline $17-4,48$ & - & - & - & - & - & \\
\hline $17-4,73-75$ & 1.34 & 2.51 & 79.0 & 60.5 & - & \\
\hline $17-4,110$ & - & - & - & - & 1.52 & \\
\hline $17-5,11$ & $=$ & $=$ & - & $=$ & 1.52 & \\
\hline $17-5,73-75$ & 1.34 & 2.53 & 78.9 & 60.2 & - & \\
\hline $17-6,40$ & - & - & - & - & - & \\
\hline $17-6,73-75$ & 1.38 & 2.57 & 76.8 & 56.9 & - & \\
\hline $17-6,90$ & - & - & - & - & 1.51 & \\
\hline $17-7,73-75$ & 1.38 & 2.59 & 77.1 & 57.1 & - & \\
\hline $17-7,102$ & - & - & - & - & 1.52 & \\
\hline $18-1,133-135$ & 1.33 & 2.59 & 80.5 & 62.0 & - & \\
\hline $18-2,31$ & - & - & - & - & - & \\
\hline $18-3,73-75$ & 1.32 & - & 85.3 & 66.4 & $=$ & \\
\hline $18-3,95$ & - & - & - & - & - & \\
\hline $18-3,140$ & - & $=$ & - & - & 1.51 & \\
\hline $18-4,73-75$ & 1.33 & 2.57 & 80.0 & 61.5 & - & \\
\hline $18-5,52$ & - & - & - & - & - & \\
\hline $18-5,73-75$ & 1.33 & 2.57 & 80.4 & 62.1 & - & \\
\hline $18-5,114$ & - & - & - & - & 1.52 & \\
\hline $18-6,33-35$ & 1.37 & 2.59 & 78.2 & 58.7 & - & \\
\hline $19-1,73-75$ & 1.38 & 2.63 & 77.6 & 57.5 & - & \\
\hline $19-2,50$ & - & - & - & - & - & \\
\hline $19-2,73-75$ & 1.37 & 2.64 & 78.7 & 58.9 & - & \\
\hline $19-2,106$ & 1.0 & - & - & - & 1.51 & \\
\hline $19-3,73-75$ & 1.38 & 2.62 & 77.6 & 57.5 & - & \\
\hline $19-4,44$ & - & $\overline{0}$ & - & - & - & \\
\hline $19-4,73-75$ & 1.39 & 2.67 & 77.9 & 57.4 & - & \\
\hline $19-4,120$ & - & - & - & - & 1.50 & \\
\hline $19-5,73-75$ & 1.44 & 2.65 & 74.3 & 52.8 & - & \\
\hline
\end{tabular}


Table 1. (Continued).

\begin{tabular}{|c|c|c|c|c|c|c|c|c|}
\hline $\begin{array}{c}\text { Sample } \\
\text { (level or interval in } \mathrm{cm} \text { ) }\end{array}$ & $\underset{\left(\mathrm{g} / \mathrm{cm}^{3}\right)}{\mathrm{eb}}$ & $\underset{\left(\mathrm{g} / \mathrm{cm}^{3}\right)}{\mathrm{e}_{\mathrm{g}}}$ & $\underset{(\%)}{\phi}$ & $\begin{array}{l}\text { Water } \\
\text { Content } \\
\text { (wt.\%) }\end{array}$ & $\begin{array}{c}V_{\mathrm{p}} \\
(\mathrm{km} / \mathrm{s})\end{array}$ & $\begin{array}{l}\text { Shear } \\
\text { Strength } \\
\left(\mathrm{g} / \mathrm{cm}^{2}\right)\end{array}$ & $\begin{array}{l}\text { Penetrability } \\
\text { (mm) }\end{array}$ & $\stackrel{k}{\left(\mathrm{~W} / \mathrm{m}^{\circ} \mathrm{K}\right)}$ \\
\hline $505-19-6,40$ & - & - & - & - & - & & & 0.87 \\
\hline $19-6,73-75$ & 1.42 & 2.60 & 75.1 & 54.4 & - & & & - \\
\hline $19-6,120$ & - & - & - & - & 1.51 & & & - \\
\hline $19-7,73-75$ & 1.40 & 2.63 & 76.4 & 55.8 & - & & & - \\
\hline $19-8,15$ & - & - & - & - & - & & & 0.87 \\
\hline $20-1,73-75$ & 1.39 & 2.61 & 76.8 & 56.5 & - & & & - \\
\hline $20-2,30$ & - & - & - & - & - & & & 0.87 \\
\hline $20-2,73-75$ & 1.37 & 2.62 & 78.5 & 58.8 & - & & & - \\
\hline $20-2,77$ & - & - & - & - & 1.52 & & & - \\
\hline $20-3,73-75$ & 1.40 & 2.58 & 75.9 & 55.6 & - & & & - \\
\hline $20-4,40$ & - & - & - & - & - & & & 0.86 \\
\hline $20-4,73-75$ & 1.37 & 2.63 & 78.4 & 58.5 & - & & & - \\
\hline $20-4,106$ & - & - & - & - & 1.51 & & & - \\
\hline $20-5,73-75$ & 1.37 & 2.56 & 77.3 & 57.7 & - & & & - \\
\hline $20-6,37$ & - & - & - & - & - & & & 0.91 \\
\hline $20-6,73-75$ & 1.43 & 2.58 & 74.0 & 53.0 & - & & & - \\
\hline $20-6,88$ & - & - & - & - & 1.51 & & & - \\
\hline $20-7,73-75$ & 1.43 & 2.51 & 73.1 & 52.5 & - & & & - \\
\hline $21-1,73-75$ & 1.43 & 2.62 & 74.6 & 53.5 & - & & & - \\
\hline $21-2,31$ & - & - & - & - & - & & & 0.90 \\
\hline $21-2,73-75$ & 1.41 & 2.62 & 75.8 & 55.1 & - & & & - \\
\hline $21-2,91$ & - & - & - & - & 1.52 & & & - \\
\hline $21-3,73-75$ & 1.42 & 2.65 & 75.4 & 54.2 & - & & & - \\
\hline $21-4,31$ & - & - & - & - & - & & & 0.87 \\
\hline $21-4,73-75$ & 1.39 & 2.61 & 76.8 & 56.5 & - & & & - \\
\hline $21-4,91$ & - & - & - & - & 1.50 & & & - \\
\hline $21-5,73-75$ & 1.41 & 2.62 & 75.9 & 55.3 & - & & & - \\
\hline $21-6,38$ & - & - & - & - & - & & & 0.99 \\
\hline $21-6,68$ & - & - & - & - & 1.50 & & & - \\
\hline $21-6,73-75$ & 1.42 & 2.63 & 75.2 & 54.2 & - & & & - \\
\hline $21-7,73-75$ & 1.43 & 2.62 & 74.9 & 53.8 & - & & & - \\
\hline $21-8,34$ & - & - & - & - & - & & & 1.02 \\
\hline $21-8,63-65$ & 1.44 & 2.62 & 74.2 & 52.9 & - & & & - \\
\hline $22-1,73-75$ & 1.53 & 2.68 & 60.6 & 46.7 & - & & & - \\
\hline $22-2,47$ & - & - & - & - & - & & & 1.09 \\
\hline $22-2,73-75$ & 1.48 & 2.68 & 72.3 & 49.9 & - & & & 1.09 \\
\hline $22-2,116$ & - & - & - & - & 1.50 & & & - \\
\hline $22-3,73-75$ & 1.51 & 2.68 & 70.7 & 48.0 & - & & & - \\
\hline $22-4,38$ & - & - & - & - & - & & & 1.08 \\
\hline $22-4,73-75$ & 1.47 & 2.65 & 72.4 & 50.3 & - & & & - \\
\hline $22-4,94$ & - & - & - & - & 1.50 & & & - \\
\hline $22-5,73-75$ & 1.48 & 2.66 & 72.4 & 50.2 & - & & & - \\
\hline $22-6,38$ & - & - & - & - & - & & & 1.00 \\
\hline $22-6,73-75$ & 1.45 & 2.64 & 73.5 & 51.9 & - & & & - \\
\hline $22-6,99$ & - & - & - & - & 1.51 & & & - \\
\hline $23-1,73-75$ & 1.45 & 2.53 & 71.5 & 50.4 & - & & & - \\
\hline $23-2,44$ & - & - & - & - & - & & & 1.05 \\
\hline $23-2,73-75$ & 1.48 & 2.65 & 72.1 & 49.9 & - & & & - \\
\hline $23-2,98$ & - & - & - & - & 1.51 & & & - \\
\hline $23-3,73-75$ & 1.46 & 2.64 & 73.1 & 51.2 & - & & & - \\
\hline $23-4,40$ & - & - & - & - & - & & & 1.04 \\
\hline $23-4,73-75$ & 1.47 & 2.66 & 73.0 & 51.0 & - & & & - \\
\hline $23-4,105$ & - & - & - & - & 1.52 & & & - \\
\hline $23-5,73-75$ & 1.50 & 2.67 & 71.3 & 48.9 & - & & & - \\
\hline $23-6,50$ & - & - & - & - & - & & & 1.07 \\
\hline $23-6,73-75$ & 1.50 & 2.66 & 70.9 & 48.5 & - & & & - \\
\hline $23-6,99$ & - & - & - & - & 1.53 & & & - \\
\hline $24-1,73-75$ & 1.48 & 2.71 & 73.2 & 50.7 & - & & & - \\
\hline $24-2,49$ & - & - & - & - & - & & & 1.08 \\
\hline $24-2,89$ & - & - & - & - & 1.51 & & & - \\
\hline $24-2,73-75$ & 1.48 & 2.71 & 72.7 & 50.1 & - & & & - \\
\hline
\end{tabular}

